\title{
1 Caulobacter requires anionic sphingolipids and deactivation of fur to lose lipid A
}

3 Justin J. Zik ${ }^{1,12}$, Sung Hwan Yoon ${ }^{2,13}$, Ziqiang Guan ${ }^{3}$, Gabriele Stankeviciute Skidmore ${ }^{4,11}$, 4 Ridhi R. Gudoor ${ }^{5,6}$, Karen M. Davies ${ }^{5,6,14}$, Adam M. Deutschbauer ${ }^{7}$, David R. Goodlett ${ }^{2,8,9}$, 5 Eric A. Klein ${ }^{4,10,11}$ and Kathleen R. Ryan ${ }^{1,7, *}$

7 'Department of Plant \& Microbial Biology, University of California, Berkeley, Berkeley, CA 94720

8 USA

$9{ }^{2}$ Department of Microbial Pathogenesis, University of Maryland School of Dentistry, Baltimore, 10 MD 21201 USA

$11{ }^{3}$ Department of Biochemistry, Duke University Medical Center, Durham, NC 27710

$12{ }^{4}$ Center for Computational and Integrative Biology, Rutgers University-Camden, Camden, NJ

1308102 USA

$14{ }^{5}$ Molecular Biosciences and Integrated Bioimaging Division, Lawrence Berkeley National

15 Laboratory, Berkeley, CA 94720 USA

$16{ }^{6}$ Department of Molecular and Cell Biology, University of California, Berkeley, Berkeley, CA

1794720 USA

$18{ }^{7}$ Environmental Genomics \& Systems Biology Division, Lawrence Berkeley National Laboratory, 19 Berkeley, CA 94720 USA

$20{ }^{8}$ Department of Biochemistry \& Microbiology, University of Victoria, Victoria, BC V8W 2Y2

21 Canada

22 'University of Victoria-Genome BC Proteomics Centre, Victoria, BC V8Z 7X8 Canada

$23{ }^{10}$ Biology Department, Rutgers University-Camden, Camden, NJ 08102 USA

$24{ }^{11}$ Rutgers Center for Lipid Research, Rutgers University, New Brunswick, NJ 08901 USA

$25{ }^{12}$ present address: Infectious Diseases Translational Research Programme, Department of

26 Microbiology and Immunology, Yong Loo Lin School of Medicine, National University of

27 Singapore, Singapore 117545, Singapore

$28{ }^{13}$ present address: National Institute of Allergy and Infectious Diseases, Bethesda, MD 20892-

290421 USA

$30{ }^{14}$ present address: Diamond Light Source Ltd. Harwell Science \& Innovation Campus, Didcot, 31 Oxfordshire, OX11 0DE, UK

$32{ }^{*}$ Correspondence: krr@berkeley.edu 
33 ORCID: Kathleen R. Ryan, 0000-0003-3657-8069; Justin J. Zik, 0000-0003-0260-2446; Eric A.

34 Klein, 0000-0003-3941-6233, Karen M. Davies, 0000-0002-3207-9337; David R. Goodlett,

35 0000-0002-8045-8200

36

37

38

39

40

41

42

43

44

45

46

47

48

49

50

51

52

53

54

55

56

57

58

59

60

61

62

63

64

65

66 
Lipid A, the membrane-anchored portion of lipopolysaccharide, is an essential component of the outer membrane (OM) of nearly all Gram-negative bacteria. Here, we identify regulatory and structural factors that together permit Caulobacter crescentus to eliminate lipid $A$ from its OM. Mutations in the ferric uptake regulator fur allow Caulobacter to survive in the absence of either LpxC, which catalyzes an early step of lipid A synthesis, or CtpA, a tyrosine phosphatase homolog which we find is needed for wild-type lipid A structure and abundance. Alterations in Fur-regulated processes, rather than iron status per se, underlie the ability to eliminate lipid A. Fitness of lipid A-deficient Caulobacter requires a previously uncharacterized anionic sphingolipid, ceramide phosphoglycerate (CPG), which also mediates sensitivity to the antibiotic colistin. Our results demonstrate that, in an altered regulatory landscape, anionic sphingolipids can support the integrity of a lipid A-deficient OM.

\section{Introduction}

Gram-negative bacteria are enclosed in a three-layer envelope, composed of the inner or cytoplasmic membrane (IM), a thin layer of peptidoglycan (PG), and an outer membrane $(\mathrm{OM})$. The $\mathrm{OM}$ is an asymmetric bilayer, with phospholipids populating the inner leaflet and lipopolysaccharide (LPS) predominating in the outer leaflet. The canonical LPS structure, first described in Escherichia coli, consists of three segments: 1) lipid A, a hexa-acylated, phosphorylated glucosamine disaccharide anchored in the membrane; 2) a core oligosaccharide usually shared by members of the same species; and 3) a repeating polysaccharide (O-antigen) which can vary highly among strains of the same species (Whitfield and Trent, 2014). LPS confers robust barrier function upon the OM, making it inherently less permeable than the IM to small hydrophobic compounds. Barrier function has been ascribed to strong lateral interactions between LPS molecules mediated by packing of the saturated acyl chains of lipid A and a network of divalent cations coordinated by negatively charged groups, particularly those on lipid A or the core polysaccharide (Nikaido, 2003).

Although the O-antigen and core polysaccharide are nearly always dispensable, it is widely accepted that the lipid A portion of LPS is essential for Gram-negative bacterial viability.

96 Some exceptions to this rule are species that possess a dual membrane system but naturally

97 lack lipid A, such as Borrelia burgdorferi, Treponema pallidum, and Sphingomonas spp.

98 (Kawahara et al., 1991; Kawasaki et al., 1994; Radolf and Kumar, 2018). Efforts to eliminate

99 lipid $A$ from $E$. coli strains have demonstrated that the intermediate molecule lipid $\mathrm{IV}_{\mathrm{A}}$ is

100 sufficient for viability, but only if the strain also has compensatory mutations that promote the 
101 export of this species across the IM (Mamat et al., 2008; Meredith et al., 2006). To date lipid A-

102 deficient mutants have been recovered in Neisseria meningitidis, Moraxella catarrhalis, and

103 Acinetobacter baumannii (Moffatt et al., 2010; Peng et al., 2005; Steeghs et al., 1998). It

104 remains unclear why at least a minimal lipid A structure is essential in some Gram-negative

105 bacteria but not others.

106 Lipid A is synthesized by the highly conserved Raetz pathway (Whitfield and Trent,

107 2014), yet significant variation exists in lipid A structures. In many species, mechanisms exist to

108 modify the 1- and 4 $\square$-phosphoryl groups of lipid A to decrease its negative charge and reduce

109 susceptibility to cationic antimicrobial peptides (Moffatt et al., 2019). In a few species,

110 replacement of the 1 - and/or $4 \square$-phosphoryl groups of lipid A with sugars is constitutive (De

111 Castro et al., 2008; Plötz et al., 2000). The predominant lipid A species in the

112 alphaproteobacterium Caulobacter crescentus (Smit et al., 2008) varies from that of E. coli

113 (Qureshi et al., 1988) in that the central glucosamine disaccharide is replaced by two 2,3-

114 diamino-2,3-dideoxy-D-glucopyranose (GlcN3N) residues, and the 1- and 4 $\square$-phosphates are

115 replaced by galactopyranuronic acid (GalpA) residues.

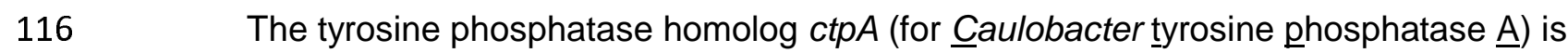

117 essential for viability and is implicated in cell envelope maintenance, but its molecular function

118 remains unknown (Shapland et al., 2011). Depletion of ctpA causes extensive OM blebbing,

119 failure to resolve PG at the division site, and cell death. Here, we show that $c t p A$ is required for

120 the wild-type structure and abundance of lipid A. A screen for suppressors of ctpA essentiality

121 recovered strains with null mutations in the O-antigen biosynthetic pathway or in the ferric

122 uptake regulator fur. Surprisingly, mutations in fur also permitted the deletion of $I p x C$, which

123 encodes an otherwise essential enzyme catalyzing the first committed step in lipid A synthesis.

$124 \Delta c t p A$ and $\triangle / p x C$ strains containing suppressor mutations have significantly reduced or

125 undetectable levels of lipid A, respectively.

126 To identify mechanisms that promote survival in the absence of lipid $A$, we used random

127 barcode transposon-site sequencing (RB-Tnseq) to identify genes important for fitness when

128 lipid A synthesis is chemically inhibited. Interestingly, we obtained several hits in genes required

129 for sphingolipid synthesis in Caulobacter (Stankeviciute et al., 2019, 2021). Since

130 Sphingomonads naturally lack LPS and bear anionic sphingolipids on the cell surface (Kawasaki

131 et al., 1994), we hypothesized that anionic sphingolipids could support viability in the

132 Caulobacter $\Delta / p x C$ strain. Indeed, we identified a previously unknown sphingolipid species,

133 ceramide phosphoglycerate, which is a critical fitness factor in the absence of lipid A. Further, 
134 we found that ceramide phosphoglycerate, rather than LPS, underlies Caulobacter's sensitivity

135 to the cationic antimicrobial peptide colistin.

\section{Results}

We used a CtpA depletion strain (see Methods, CtpA depletion strain) to identify mutations that would support Caulobacter viability in the absence of CtpA (Shapland et al., 2011). When depleting CtpA by transferring cells from xylose-supplemented rich medium (PYEX) to dextrose-supplemented rich medium (PYED), KR3906 exhibits division defects, significant OM blebbing, and death (Fig. 1). We UV-mutagenized KR3906, plated survivors on solid PYED medium to deplete CtpA, and grew the recovered colonies in PYED liquid medium to allow loss of the plasmid bearing $\operatorname{ctp} A$. Isolates that had successfully lost the plasmid were identified via chloramphenicol sensitivity and the inability to PCR-amplify $\operatorname{ctpA}$ (Fig. 1A). Genome resequencing of 17 confirmed suppressors yielded 15 strains with mutations in nine genes predicted to participate in O-antigen biosynthesis, one strain with a single mutation in fur, encoding the ferric uptake regulator, and one strain harboring a mutation in fur along with a mutation in an O-antigen biosynthetic gene (Table S1). Due to the frequent occurrence of frameshift or nonsense mutations, we assumed that each mutation disrupted the function of the affected gene.

We chose the following candidate suppressors for further analysis: (i) CCNA_00497, encoding a predicted rhamnosyl transferase necessary for wild-type levels of O-antigen containing smooth LPS (S-LPS) (Hershey et al., 2019); (ii) CCNA_01553, encoding a glycosyltransferase which initiates O-antigen synthesis on undecaprenyl-phosphate (Toh et al., 2008); (iii) CCNA_03733, encoding a homolog of manC involved in synthesizing the activated

158 sugar GDP-D-mannose (Samuel and Reeves, 2003), which is present in the core

159 oligosaccharide and O-antigen of Caulobacter S-LPS (Jones et al., 2015); and iv)

160 CCNA_00055, encoding the iron-responsive transcriptional regulator Fur (da Silva Neto et al.,

161 2013). We individually deleted these genes in the CtpA depletion strain KR3906 while

162 propagating the strains on PYEX.

163 To determine how each deletion affects cells during acute CtpA depletion, we shifted 164 each mutant onto liquid or solid PYED medium and observed cell morphology and viability (Fig.

165 1). Compared to CtpA depletion in KR3906, acute depletion of CtpA in the $\triangle$ fur mutant caused 166 much less OM blebbing, but still yielded elongated cells indicative of a division defect (Fig. 1C).

167 Surprisingly, neither OM blebbing nor cell chaining/elongation was markedly improved when 
168 CtpA was depleted from the strains lacking CCNA_00497, CCNA_01553, or CCNA_03733.

169 Despite the persistence of one or more morphological defects, deletion of fur, CCNA_01553, or

170 CCNA_03733 significantly improved cell viability during depletion of CtpA on solid PYED

171 medium (Fig. 1B). In contrast, the deletion of CCNA_00497 only weakly improved survival on

172 PYED medium. Two independent point mutations in CCNA_00497 were isolated in the

173 suppressor screen, but each mutant also harbored 1-2 other mutations (Table S1) that may

174 have contributed to the fitness of the original isolates.

175 To acquire stable $\triangle \operatorname{ctp} A$ derivatives of each suppressor strain constructed above, we

176 plated each new mutant on PYED medium and screened viable colonies for loss of the ctpA-

177 bearing plasmid. The OM of each stable $\Delta c t p A$ strain was smooth with minimal blebbing, but

178 chains of cells were still prevalent in the $\triangle C C N A \_01553$ and $\triangle$ fur mutants (Fig. 1D). These

179 reconstituted suppressor strains are morphologically similar to the original isolates containing

180 point mutations in the same genes (Fig. S1A). Suppressed $\Delta c t p A$ mutants grow more slowly

181 than the wild-type strain NA1000 and the corresponding $\operatorname{ctp} A^{+}$strains, but all achieve similar

182 stationary phase densities in PYE medium (Fig. S1B). As expected, restoring the expression of

183 fur, CCNA_00497, or CCNA_03733 using a xylose-inducible promoter reduced the viability of

184 each corresponding stable $\triangle c t p A$ strain (Fig. S1C). Thus, null mutations affecting fur or Oantigen biosynthesis allow Caulobacter to survive in the absence of $\operatorname{ctp} A$.

To confirm the functions of suppressor genes predicted to be involved in O-antigen synthesis, we deleted the individual genes in a control strain lacking $s s p B$ (see Methods, CtpA depletion strain). Whole-cell lysates treated with proteinase $\mathrm{K}$ were probed with antibodies recognizing S-LPS (Fig. S2A) or stained with Pro-Q Emerald 300 to detect carbohydrates (Fig. S2B). As previously observed, a control strain $\triangle C C N A \_01068$ (wbqA) and the $\triangle C C N A \_01553$ mutant lacked S-LPS (Awram and Smit, 2001), which migrates as a single high-molecular weight species in Caulobacter (Walker et al., 1994). Mutations in CCNA_00497 or CCNA_03733 caused partial or complete elimination of S-LPS, respectively. S-LPS was

194 restored to each mutant by xylose-driven complementation of the respective genes (Fig. S2C).

195 In contrast to strains with mutations in CCNA_00497, CCNA_01553, or CCNA_03733, the $\triangle$ fur $196 \Delta s s p B$ mutant contained wild-type levels of S-LPS (Fig. S2A-C), indicating that fur mutations do 197 not suppress the lethality of $\triangle c t p A$ by eliminating the O-antigen.

198 We propose that a low-molecular weight band in most lysates represents lipid A + core 199 polysaccharide (Fig. S2B and S2C, ${ }^{* *}$ ). This inference is supported by the band pattern in the $200 \triangle C C N A \_03733$ (manC) mutant. The core oligosaccharide of Caulobacter LPS contains a single 
201 penultimate mannose residue (Jones et al., 2015); thus, the reduced size of the indicated band

202 for $\triangle C C N A \_03733$ (Fig. S2B and S2C, *) may arise from an incomplete core oligosaccharide.

Suppressor mutations permit $\triangle \mathrm{ctpA}$ and $\Delta \mathrm{lpxC}$ strains to survive with little or no lipid $A$

$\operatorname{ctp} A$ is transcribed divergently from an operon containing $m s b A, I p x J, k d t A$, and $I p x K$ (Zhou et al., 2015), which in other bacteria participate in the synthesis and export of lipid A + core polysaccharide (Whitfield and Trent, 2014). Like ctpA, these genes are essential for Caulobacter viability (Christen et al., 2011). Since CtpA depletion results in OM defects, and suppressor mutations were identified in O-antigen biosynthetic genes, we hypothesized that $c t p A$ is required for some aspect of LPS synthesis or export.

We performed hot aqueous-phenol extraction of LPS from suppressor mutants lacking $\operatorname{ctp} A$, along with their $\operatorname{ctp} A^{+}$counterparts, and analyzed them by PAGE and Pro-Q Emerald 300 staining. Full-length S-LPS was recovered from NA1000, $\Delta s s p B$, and $\Delta$ fur $\Delta s s p B$ (Fig. 2B, ${ }^{\star * \star}$ ), whereas only smaller LPS species were present in the $\triangle s s p B$ strains lacking CCNA_00497, CCNA_01553, or CCNA_03733. Interestingly, all $\triangle c t p A$ strains harboring suppressor mutations were deficient in low-molecular weight species that could represent lipid A +/- core oligosaccharide (Fig. 2B). We therefore used the Limulus Amebocyte Lysate ( $L A L)$ assay to measure lipid $A$ abundance in live Caulobacter strains. The stable $\Delta c t p A$ mutants contained approximately 1,000-fold less lipid A than NA1000 or the corresponding $c t p A^{+}$strains (Fig. 2A). A, we asked if mutations in fur or O-antigen synthesis could allow Caulobacter to lose lipid A entirely. LpxC catalyzes the first committed step in lipid A synthesis, removal of the 2-acetyl group from acylated UDP-GlcNAc (Whitfield and Trent, 2014). The IpxC homolog CCNA_02064

224 is essential for viability in wild-type Caulobacter (Christen et al., 2011). We constructed an LpxC 225 depletion strain (KR4007) analogous to the CtpA depletion strain. We subsequently deleted fur, 226 CCNA_00497, CCNA_01553, or CCNA_03733 in this strain and examined the effects of acute

227 LpxC depletion during growth in PYED. In the absence of candidate suppressor mutations,

228 LpxC depletion yielded chains of cells with extensive membrane blebs. Cells lacking a gene for 229 O-antigen synthesis still showed membrane blebs and chaining when LpxC was depleted (Fig. 230 3A). Cells lacking fur had far fewer OM blebs upon LpxC depletion but were still frequently 231 elongated or chained (Fig. 3A). These morphologies are generally similar to those seen during 232 CtpA depletion, but unlike CtpA, only $\triangle$ fur allowed significant growth of the LpxC depletion strain 233 on solid PYED medium (Fig. 3B). 
When we attempted to isolate stable $\Delta / p x C$ mutants using the same outgrowth and screening process as for $\triangle c t p A$, only the strain harboring a $\Delta$ fur mutation permitted complete loss of $I p x C$. We initially recovered two stable $\Delta / p x C$ isolates from a depletion strain harboring $\triangle C C N A \_00497$, but genome resequencing revealed that these strains had acquired additional mutations in fur (Table S2). As in $\Delta c t p A \Delta f u r \Delta s s p B$, the stable $\Delta l p x C \Delta f u r \Delta s s p B$ mutant still formed chains (Fig. 1D), and xylose-driven fur expression induced lethality in this background (Fig. 3C).

Background levels of lipid $A$ were detected in $\Delta / p x C \Delta$ fur $\Delta s s p B$ cells in the LAL assay

242 (Fig. 2A), strongly suggesting that lipid $A$ is absent. To corroborate this result, we extracted LPS 243 species by three distinct methods, separated them by PAGE, and stained with Pro-Q Emerald 244 300. Hot aqueous-phenol extracts of $\Delta / p x C \Delta$ fur $\Delta s s p B$ cells were deficient in S-LPS and 245 putative lipid A +/- core species (Fig. 2B). However, unknown carbohydrate-containing species were extracted by this method. Extraction of free lipid A (El Hamidi et al., 2005) revealed that a species of $\sim 1800 \mathrm{Da}$, consistent with the mass of Caulobacter lipid A (Smit et al., 2008), is present in NA1000 but absent from $\Delta l p x C \Delta$ fur $\Delta s s p B$ (Fig. 2C, left). Again, however, unidentified carbohydrate species were present in these extracts. Lastly, the method of Darveau and Hancock (Darveau and Hancock, 1983) yielded a single rough LPS species which was present in NA1000 and absent from $\Delta / p x C \Delta$ fur $\Delta s s p B$ (Fig. 2C, right); this method resulted in no unidentified contaminants. Although some Caulobacter extracts contain unidentified lipids, these assays together strongly imply that lipid $\mathrm{A}$ is absent from the $\Delta / p x C \Delta f u r \Delta s p B$ mutant. Xylose-driven expression of $\operatorname{IpxC}$ or $\operatorname{ctp} A$ restored the production of lipid A-containing species to $\Delta / p x C \Delta$ fur $\triangle s s p B$ or $\triangle \operatorname{ctp} A \triangle C C N A \_01553 \Delta s s p B$, respectively (Fig. 2D).

Lipid A extracts from $\Delta \operatorname{ctp} A \Delta$ fur $\Delta s s p B, \Delta / p x C \Delta$ fur $\Delta s s p B$, and control strains were further analyzed by matrix-assisted laser desorption/ionization tandem mass spectrometry (MALDI-MS/MS). Wild-type NA1000, $\Delta s s p B$, and $\Delta$ fur $\Delta s s p B$ extracts contained predominantly the full-length lipid $A$ ( $m / z$ 1874, (Smit et al., 2008) and lesser amounts of an ion at $m / z 1858$ that differs from 1874 by $16 \mathrm{~m} / z$, consistent with the absence of one hydroxyl group (Fig. S3AC). MALDI-MS analyses of lipid A extracts from $\Delta \operatorname{ctp} A \Delta$ fur $\triangle s s p B$ cells revealed no ions consistent with full-length Caulobacter lipid A, but identified ions at $m / z 1682$ and $m / z 1486$ (Fig. S3D) that appeared to be missing the GalpA residues at the 1 and $4 \square$ positions. Tandem MS

265 for canonical lipid A structures. Although additional characterization is needed, our results 266 suggest that Caulobacter mutants lacking CtpA produce an incomplete lipid A species which 267 retains phosphate at the 1 and $4 \square$ positions, and which lacks one or more of the secondary 
268 phospholipids. While these incomplete lipid A species were detectable by mass spectrometry,

269 gel electrophoresis and LAL assays indicate that they are much less abundant than the lipid $A$ in 270 wild-type strains.

$271 \quad$ Lipid A extracts from the $\Delta / p x C \Delta$ fur $\Delta s s p B$ mutant yielded no ions consistent with wild-

272 type lipid $A$ and instead contained an unknown lipid (Fig. S3E, $m / z$ 1412). Numerous attempts

273 to interpret the structure of this ion using the same type of tandem MS data as used in Fig. S3A-

274 D failed to generate a structural hypothesis resembling obvious choices such lipid A derivatives

275 or cardiolipin. Again, it is important to note that while this unknown ion was detected by mass

276 spectrometry, gel electrophoresis and $L A L$ assays together indicate that lipid $A$ is absent from

$277 \Delta / p x C \Delta$ fur $\Delta s s p B$ cells.

Lipid A-deficient Caulobacter mutants produce a three-layer cell envelope

We analyzed NA1000, $\Delta c t p A \Delta f u r \Delta s s p B$ and $\Delta l p x C \Delta$ fur $\Delta s s p B$ strains via electron cryotomography to assess the effects of mutations on cell envelope structure (Movies S1-S4). As expected, the S-layer is absent from both mutants due to the loss of its O-antigen attachment site (Walker et al., 1994). Despite drastic reductions in lipid A levels, the $\Delta c t p A \Delta f u r$ $\triangle s s p B$ and $\Delta / p x C \Delta$ fur $\Delta s s p B$ mutants still generate a three-layer cell envelope, including an OM (Fig. 2E). Although much less severe than during acute CtpA depletion (Movie S4), membrane blebs were often observed at the cell poles or division sites in $\triangle \operatorname{ctp} A \Delta f u r \Delta s s p$ and $\Delta / p \times C \Delta$ fur $\Delta s s p B$ cells (Fig. 2E). A significant proportion of $\operatorname{ctp} A$ and $I p \times C$ mutant cells exhibited defects in stalk structure or IM distortions at the pole or midcell $(\mathrm{N}=100 ; \Delta c t p A \Delta$ fur $\Delta s s p B: 61 \% ; \Delta l p x C \Delta$ fur $\Delta s s p B$ : 51\%; NA1000: 4\%).

292 lipid $A$ Mutations in fur or O-antigen synthesis genes did not appreciably increase chemical sensitivity compared to NA1000, while strains lacking $\operatorname{ctp} A$ or IpxC had greater sensitivity to a subset of antibiotics and to all tested detergents (Fig. 4A). In sharp contrast, the $\Delta / p x C \Delta$ fur $\Delta s s p B$ strain and $\Delta \operatorname{ctp} A \Delta s s p B$ strains with suppressor mutations were much less susceptible to CHIR-090,

$299 \triangle \operatorname{ctp} A$ mutants are relatively insensitive to $\mathrm{CHIR}-090$ because they already produce little lipid $\mathrm{A}$ 300 or lack the target enzyme. 
In agreement with its ability to suppress the lethality of $\Delta / p x C$ and $\triangle \operatorname{ctp} A$ mutations, the $\Delta$ fur allele by itself greatly reduced the sensitivity of Caulobacter to CHIR-090 (Fig. 4B). Fur senses available $\mathrm{Fe}^{2+}$ by reversibly binding a [2Fe-2S] cluster (Fontenot et al., 2020). We therefore asked whether iron limitation could mimic the phenotypes of a $\Delta f u r$ mutant. Culturing NA1000 with the iron chelator 2,2'-dipyridyl reduced its susceptibility to CHIR-090 to match that of the $\Delta$ fur mutant (Fig. 4B). Neither depleting LpxC in fur ${ }^{+}$cells nor inducing fur in $\Delta / p x C \Delta f u r$ $\triangle \operatorname{ssp} B$ cells caused a reduction in viability in the presence of 2,2'-dipyridyl (Fig. 4C, D). The NA1000, $\Delta s s p B$, and $\Delta$ fur $\Delta s s p B$ strains cultured in 2,2'-dipyridyl retained LPS and lipid A +/core species (Fig. 4E). Therefore, low iron availability does not induce the loss of lipid $A$, but is sufficient to maintain Caulobacter viability when lipid A is eliminated by chemical or genetic means.

In diverse bacteria, Fur inhibits the expression of iron uptake systems and promotes the expression of proteins that utilize iron, contributing to iron homeostasis (Andrews et al., 2013). Because they are impaired in iron sensing, fur mutants of other bacteria accumulate more available iron than the corresponding wild-type strains (Liu et al., 2020; Wofford et al., 2019). We measured available iron levels using a streptonigrin (SNG) sensitivity assay (Justino et al., 2007; Nachin et al., 2001), because SNG killing is linked to the intracellular formation of oxygen radicals in the presence of iron (Hassett et al., 1987; Yeowell and White, 1982). Growth of the $\Delta$ fur and $\Delta$ fur $\Delta s s p B$ strains was almost completely inhibited by $0.25 \mu \mathrm{g} / \mathrm{ml}$ SNG, while NA1000 was only mildly inhibited (Fig. 4F), consistent with higher levels of available iron in $\Delta$ fur mutants.

321 These findings indicate that both excess available iron (in fur mutants) and iron depletion (by

322 2,2-dipyridyl) are compatible with the elimination of lipid A. Since fur deletion and iron chelation

323 have the same effect on Fur-regulated gene expression, but opposite effects on Fur-

324 independent iron signaling (Fig. 4G), this implies that iron-mediated processes regulated by Fur

325 are specifically responsible for the survival of lipid A-deficient Caulobacter (Leaden et al., 2018;

326 da Silva Neto et al., 2013).

$R B$-Tnseq identifies sphingolipid synthesis genes needed for fitness when lipid A synthesis is chemically inhibited

To identify additional factors that promote the survival of lipid A-deficient Caulobacter, we challenged an RB-Tnseq library constructed in NA1000 (Price et al., 2018) with CHIR-090.

332 Individual barcode frequencies were measured by high-throughput sequencing before each trial 333 and after growth in either PYE or PYE $+2 \mu \mathrm{g} / \mathrm{ml}$ CHIR-090. To identify genes that are

334 particularly important when $L p x C$ is inhibited, we averaged and compared the gene fitness 
scores (Wetmore et al., 2015) from three trials in each condition (Fig. 5A). We anticipated that mutations in fur would increase fitness in CHIR-090, but the NA1000 RB-Tnseq library contained no insertions in fur. Surprisingly, nearly all genes known to be regulated by Fur (Leaden et al., 2018; da Silva Neto et al., 2013) had similar fitness scores in unstressed and CHIR-090-exposed cultures (Fig. 5A).

Focusing on genes whose average fitness scores were $\geq 1$ point lower in CHIR-090-

341 treated cultures than in control cultures, we identified five genes involved in sphingolipid

342 synthesis: spt (CCNA_01220), acyl-carrier protein (acp, CCNA_01221), cerR (CCNA_01222),

343 ACP-synthetase (acps, CCNA_01223), and bcerS (CCNA_01212) (Olea-Ozuna et al., 2021;

344 Stankeviciute et al., 2021). Additionally, fitness scores were lower for transposon insertions in a 345 neighboring operon of three uncharacterized genes predicted to modify lipids (CCNA_01217-

346 01219, Marks et al., 2010) (Fig. 5A). To examine the roles of genes in the uncharacterized operon, we constructed unmarked deletions in the NA1000 and $\Delta$ fur $\Delta s s p B$ backgrounds and complemented them with the corresponding genes expressed from the inducible vanA promoter (Thanbichler et al., 2007). Loss of spt, CCNA_01217, CCNA_01218, or CCNA_01219 greatly increased the susceptibility to CHIR-090, either in NA1000 or in $\Delta$ fur $\Delta s s p B$ cells (Fig. 5B), and expression of the complementing gene from the vanA locus restored the wild-type level of susceptibility, validating the RB-Tnseq results.

Mutations in CCNA_01217-01219 or spt could increase CHIR-090 sensitivity via distinct mechanisms: by damaging the cell's permeability barrier and giving easier access to CHIR-090, by making it more difficult for cells to grow after lipid A synthesis is inhibited, or both. To eliminate changes in drug access as a factor in the experiment, we measured the effects of CCNA_01217, CCNA_01218, and spt upon cell viability when LpxC was depleted. We deleted individual genes in the strain $\Delta / p x C \Delta f u r \Delta s s p B+P x y l-l p x C:: 3 x F L A G-s s r A(K R 4091)$ and complemented them with vanA-driven copies as described above. The parent strain lacks fur and grows in PYED medium when LpxC is depleted. In contrast, KR4091 lacking CCNA_01217 or spt grew poorly in PYED medium, and growth in PYED was fully or partially restored by

362 expressing the complementing gene from the vanillate promoter (Fig. 5C). KR4091 lacking 363 CCNA_01218 grew poorly in PYEX and PYED media without vanillate, indicating that KR4091 364 requires CCNA_01218for fitness even when LpxC is produced. Growth was improved by 365 vanillate-driven expression of CCNA_01218 (Fig. 5C). Since this assay does not rely on an 366 exogenous chemical, we conclude that CCNA_01217-8 and spt are critical for the fitness of lipid 367 A-deficient Caulobacter, not simply for the exclusion of CHIR-090. 
CCNA_01217-01219 convert neutral ceramide to an anionic sphingolipid, ceramide phosphoglycerate

The importance of Spt for viability in the absence of lipid A indicated a role for

372 sphingolipids in this phenotype. Since Sphingomonads produce anionic glycosphingolipids

373 (GSLs) on the outer membrane (Kawasaki et al., 1994), we initially hypothesized that

374 Caulobacter responds to IpxC deletion by upregulating GSL production. However, neither

375 Caulobacter sphingolipid glycosyltransferase (sgt1 or sgt2, Stankeviciute et al., 2019) was

376 important for the fitness of CHIR-090-treated cells. A careful analysis of the Caulobacter

377 lipidome revealed a previously unidentified sphingolipid species, ceramide phosphoglycerate

378 (Fig. 6A). In fact, we identified two forms of this lipid containing either one or two

379 phosphoglycerate moieties (Fig. 6A) that we have designated CPG and CPG2. LC/MS/MS analysis confirmed the proposed structures of these lipids (Fig. 6B).

To determine whether CCNA_01217-01219 are involved in CPG/CPG2 synthesis, we analyzed lipid extracts from mutant and complemented mutant strains. Deletion of CCNA_01217 resulted in the loss of CPG2 but had no effect on CPG (Fig. 6C). CCNA_01217 has a conserved phosphatidylglycerophosphate synthase (PgsA) domain which is normally involved in phosphatidylglycerol (PG) synthesis. PG is the dominant phospholipid in Caulobacter (Stankeviciute et al., 2019), but the essential PgsA ortholog CCNA_03002 is likely responsible for PG synthesis (Christen et al., 2011; Marks et al., 2010). Thus, we conclude that CCNA_01217 adds the second phosphoglycerate to form CPG2. Deletion of CCNA_01218 led to the loss of CPG, CPG2 and ceramide-phosphate (Fig. 6C). CCNA_01218 is annotated as a sphingosine kinase-related protein and has a conserved LCB5 domain (Nagiec et al., 1998). Therefore, we propose that CCNA_01218 adds the initial phosphate on the ceramide. Lastly, the $\triangle C C N A \_01219$ mutant lost CPG and CPG2 but retained ceramide-phosphate (Fig. 6C). This is consistent with CCNA_01219 adding a glycerate molecule to ceramide-phosphate to form CPG. CCNA_01219 has no conserved domains, and a BLAST analysis identified homologs only in Caulobacterales and Sphingomonodales. Each deletion could be complemented by expressing the respective gene from a vanillate-inducible promoter (Fig. 6C). Based on these data, we propose a mechanism where CCNA_01218 (CpgB)

398 phosphorylates ceramide, CCNA_01219 (CpgC) adds a glycerate, and CCNA_01217 (CpgA) 399 adds a second phosphoglycerate (Fig. 6D). We note that the amount of CPG/CPG2 detected 400 appears to be a relatively small percentage of the total lipidome (Fig. S4), raising the question 401 of how these lipids can enable survival in the absence of lipid A. The CPG2 molecule is very 
polar, as evidenced by its very long LC retention time, and we expect that this lipid is not efficiently extracted by standard methods. Though we tried several modifications to increase the extraction yield, we made only marginal improvements. Additionally, our genetic data show that CpgA adds the second phosphoglycerate molecule to generate CPG2, but we cannot rule out the possibility of higher-order polymers containing additional phosphoglycerates, which would

407 be even more polar and difficult to extract.

\section{Ceramide phosphoglycerate mediates susceptibility to colistin}

Cationic antimicrobial peptides (CAMPs) have been demonstrated to kill Gram-negative bacteria by first interacting with negatively charged groups on surface-exposed LPS.

412 Phosphates at the 1 and $4 \square$ positions of lipid $A$ are particularly important for this interaction, and 413 several bacteria possess mechanisms to modify them, reducing their negative surface charge 414 and sensitivity to CAMPs (Moffatt et al., 2019; Velkov et al., 2010). Despite lacking phosphate 415 groups on its lipid A, Caulobacter is highly sensitive to colistin (Fig. 7A) and the antimicrobial 416 effect is retained in the lipid A-deficient strain $\Delta / p x C \Delta$ fur $\Delta s s p B$ (Fig. 7A). Since the CPG/CPG2 417 lipids are anionic, we considered whether they may be the colistin target in Caulobacter. Indeed, 418 the growth of mutants lacking $c p g A, c p g B$, or $c p g C$ was unaffected by colistin (Fig. 7A). Since 419 the deletion of $\operatorname{cpg} A$, catalyzing the conversion of CPG to CPG2, can alone greatly reduce 420 colistin sensitivity, and since the elimination of lipid A had no effect, we infer that a primary 421 target of colistin on the Caulobacter surface is CPG2. Furthermore, these findings are 422 consistent with our hypothesis that CPG lipids are a significant component of the OM whose 423 detection is limited by inefficient extraction.

\section{Discussion}

426 CtpA is required for wild-type lipid A structure and abundance

427 We performed a suppressor screen to discover the essential function of CtpA, which 428 contains active site residues characteristic of tyrosine phosphatases. Inactivation of fur or genes 429 involved in O-antigen synthesis permitted the deletion of $\operatorname{ctp} A$ and yielded cells with drastically 430 reduced amounts of lipid $\mathrm{A}$. MS/MS analysis of the remaining lipid A extracted from $\Delta c t p A \Delta$ fur $431 \triangle s s p B$ were consistent with species that retain phosphoryl groups at the 1 and $4 \square$ positions of 432 the central disaccharide, suggesting that CtpA is responsible for dephosphorylating at least one 433 of these positions, in preparation for the addition of GalpA residues.

434 Some alphaproteobacteria produce lipid A species with a tri- or tetrasaccharide 435 backbone (De Castro et al., 2008). In Rhizobia, the phosphatases LpxE and LpxF 
436 dephosphorylate the 1 and $4 \square$ positions, respectively, of lipid $A$ at the periplasmic surface of the

437 IM (Karbarz et al., 2003; Wang et al., 2006). Sugars are then added to the 1 and $4 \square$ positions

438 by the glycosyltransferases RgtF and RgtD, respectively, before the transport of mature LPS

439 molecules to the OM (Brown et al., 2012, 2013). NA1000 harbors a gene (CCNA_03113) with

440 similarity to LpxE, but none with similarity to LpxF, raising the possibility that CtpA substitutes

441 for LpxF. Additional work is required to verify this model; however, dephosphorylation of a lipid

442 A intermediate by CtpA could explain the ability of mutations in O-antigen synthesis to suppress

$443 \triangle \operatorname{ctp} A$. If the retention of 1- and 4 $\square$-phosphates impairs lipid $A$ trafficking, then removal or

444 reduction of the O-antigen could partially compensate and allow at least a small amount of

445 structurally altered lipid A to reach the cell surface.

The elimination of lipid $A$ in Caulobacter crescentus requires a novel anionic sphingolipid

Here we demonstrate that the enzyme $L p x C$ and lipid $A$ itself are dispensable for viability

449 in Caulobacter crescentus, conditional upon the absence of Fur and the presence of a

450 previously uncharacterized anionic sphingolipid, ceramide phosphoglycerate (Fig. 7B). LPS

451 molecules form a robust permeability barrier based on 1) tight packing of the six saturated acyl

452 chains of lipid $A$, and 2) a lateral network formed by the bridging of phosphate groups on lipid $A$

453 or the core polysaccharide by divalent cations such as $\mathrm{Mg}^{2+}$ and $\mathrm{Ca}^{2+}$ (Nikaido, 2003).

454 Caulobacter lipid A and core polysaccharide lack the phosphates that would participate in a 455 lateral network with divalent cations (Smit et al., 2008). We propose that negative charges on

$456 \mathrm{CPG} / \mathrm{CPG} 2$ can provide this function in the Caulobacter OM, accounting for the observation that $457 \quad \operatorname{cpg} A B C$ and other sphingolipid synthesis genes are important for fitness even in non-stress 458 conditions (Fig. 5A and Christen et al., 2011). Evidence that CPG/CPG2 contribute negative 459 charge to the Caulobacter OM comes from studies of CAMP sensitivity. We previously observed 460 that Spt is necessary for susceptibility to polymyxin B, but Sgt1 and Sgt2, which convert neutral 461 ceramide to the anionic glycosphingolipid GSL-2, are not required (Stankeviciute et al., 2019).

462 This result was puzzling, because neutral ceramide was not expected to be a target for CAMP

463 activity. Here we provide an explanation by showing that neutral ceramide is converted by

$464 \mathrm{Cpg} A B C$ to a different anionic species, CPG2, and that this lipid, rather than LPS, is critical for 465 colistin susceptibility.

467 Inhibition of Fur-mediated gene expression is necessary to survive in the absence of lipid $A$ 468 In contrast to Sphingomonads, the presence of cell surface sphingolipids is not sufficient 469 for Caulobacter to survive in the absence of lipid A. Instead, the iron-responsive transcription 
470 factor Fur must also be deactivated. Both iron limitation (via growth in 2,2-dipyridyl) and excess

471 available iron (due to a disruption in iron homeostasis in $\Delta$ fur) supported the viability of lipid A-

472 deficient Caulobacter. These results imply that genes or processes regulated by Fur in complex

473 with iron, rather than those regulated by iron independently of Fur, are the critical factors.

$474 \quad$ Fur controls iron homeostasis in Caulobacter by directly or indirectly regulating $\sim 120$

475 genes in combination with iron (Leaden et al., 2018; da Silva Neto et al., 2009, 2013). A

476 significant fraction of the Fur regulon, comprising 45 genes, is predicted to encode membrane

477 proteins functioning in transport reactions or energy metabolism. Caulobacter Fur represses the

478 transcription of genes predicted to mediate iron uptake, and it activates the expression of genes

479 encoding iron-containing enzymes, including respiratory complexes containing Fe/S clusters or

480 heme groups. Fur is linked to oxygen signaling in Caulobacter by activating the transcription of

481 fixK, which mediates the response to hypoxia (Crosson et al., 2005). In addition, the $\Delta$ fur mutant

482 has a constitutively elevated level of intracellular oxidation, implicating Fur in the prevention of

483 oxidative stress (Leaden et al., 2018).

$484 \quad$ Our ctpA suppressor screen retrieved mutations in fur, but not in genes whose

485 transcription is activated by Fur. Thus, there is unlikely to be a singular Fur-regulated gene

486 whose expression prevents the elimination of lipid A. Consistently, RB-Tnseq revealed that no

487 transposon insertions in Fur-activated genes led to significantly increased fitness during

488 challenge with CHIR-090. Since suppressor mutations would be more likely to cause loss than

489 gain of function, we might not retrieve suppressors that work by increasing gene expression or

490 activity. However, if there were a single Fur-repressed gene whose upregulation was critical for

491 the ability to lose lipid $A$, then transposon insertions in this gene would be expected to reduce

492 the fitness of CHIR-090-treated cells. Again, no individual gene fits this profile, but one caveat is 493 that essential genes are excluded from RB-Tnseq analysis.

$494 \quad$ Based on our genetic data, mutations in fur could support the viability of lipid A-deficient

495 Caulobacter via 1) downregulation of multiple Fur-activated genes, 2) upregulation of multiple

496 Fur-repressed genes, and/or 3) activation of compensatory cellular stress responses. Since Fur

497 regulates the expression of many OM and IM proteins, deletion of fur could alter envelope

498 composition in a manner that renders lipid A non-essential. Alternatively, the transcriptional

499 changes and oxidative stress which follow from fur deletion could activate a network of stress

500 responses which together make it possible to eliminate lipid $A$.

501

502 The search for principles governing lipid A essentiality 
Hypotheses to explain the essential nature of lipid A include its chemical barrier function, the detrimental activation of stress responses when it is depleted, its role in OM protein biogenesis or function, and its mechanical role in resisting turgor pressure (Rojas et al., 2018; Zhang et al., 2013). Caulobacter is only the fourth LPS-bearing Gram-negative bacterium

507 demonstrated to survive in the absence of lipid A, following N. meningitidis, M. catarrhalis, and

508 A. baumannii. So far, however, no single theme has emerged to explain why this select and

509 phylogenetically diverse group of Gram-negative species is capable of surviving without lipid A.

In A. baumannii, proteins which synthesize PG in lateral cell walls (the elongasome) are critical for the fitness of lipid A-deficient strains, suggesting that alterations in PG structure are needed to compensate for the OM's loss of mechanical strength (Simpson et al., 2021). However, since elongasome components are essential for viability in wild-type Caulobacter (Christen et al., 2011), RB-Tnseq could not reveal their fitness effects in CHIR-090-treated cultures. Lipid A-deficient strains of $A$. baumannii consistently display increases in the expression of lipoproteins and the Lol pathway for lipoprotein transport to the OM (Boll et al., 2016; Henry et al., 2015). Two lipoprotein synthesis genes, Igt (CCNA_00525) and Int (CCNA_00050), had markedly reduced fitness scores in CHIR-090-treated Caulobacter cultures compared to unstressed cultures, so OM lipoproteins may help to compensate for the absence of lipid $A$ in diverse species.

A. baumannii $\triangle I p x C$ mutants have growth and morphological defects that are corrected

522 when the growth rate is limited by environmental factors such as low temperature or nutrient 523 limitation (Nagy et al., 2019), suggesting that one barrier to the elimination of lipid $A$ is the rate 524 of synthesis of alternative molecules to constitute the OM. Although $\Delta$ fur slows the growth of

525 Caulobacter (Fig. S1, Table S3, and da Silva Neto et al., 2009), we found that slow growth in

526 PYE medium at a reduced temperature was not sufficient to support the viability of fur ${ }^{+}$

527 Caulobacter depleted of LpxC (Fig. S5A), or of $\Delta / p x C \Delta$ fur $\Delta s s p B$ cells with fur expression

528 restored (Fig. S5B). The $\Delta$ fur mutation therefore provides a benefit beyond decreasing the 529 growth rate, which remains to be elucidated.

$530 \quad$ Our work suggests that possession of genes to produce anionic sphingolipids may

531 provide certain bacteria with a unique pathway to the elimination of lipid A. In addition to

532 facilitating OM remodeling, anionic sphingolipids can also underlie clinically important

533 phenotypes, even in wild-type membranes that retain LPS, such as the susceptibility to CAMPs,

534 which are used as a last line of defense against multidrug-resistant bacterial infections. Thus,

535 functions traditionally attributed to lipid A may be performed wholly or in part by alternative 
536 lipids, underscoring the need to study lipid A functions in diverse species and to identify and

537 functionally characterize novel lipids.

538

\section{Acknowledgments}

The authors thank Charlie Huang, Morgan Price, Laura Herron and Henry Seaborn for

542 instrumentation of Lawrence Berkeley National Laboratory and the Bay Area CryoEM resource,

543 maintained by Jonathan Remis and Daniel Toso respectively. Assistance in Linux computer

544 maintenance was provided by Paul Tobias. D.R.G. thanks the International Centre for Cancer

545 Vaccine Science project of the International Research Agendas program of the Foundation for

546 Polish Science co-financed by the European Union under the European Regional Development

547 Fund MAB/2017/03 at the University of Gdansk. Funding for this work was provided by National

548 Science Foundation awards 1553004 and 2031948 to E.A.K. and 1615287 to K.R.R., and

549 National Institutes of Health awards GM111066-01 and 1R01Al123820-01 to D.R.G.

550 Cryoelectron tomography work was supported by the Laboratory Directed Research and

551 Development Program of Lawrence Berkeley National Laboratory under U.S. Department of

552 Energy Contract No. DE-AC02-05CH11231.

554 Author contributions

555 J.J.Z., E.A.K., and K.R.R. conceived the project. Mass spectrometry experiments were

556 performed and analyzed by S.H.Y., D.R.G., and Z.G. Electron cryotomography experiments

557 were performed and analyzed by R.G. and K.M.D. RB-Tnseq experiments, sequencing, and

558 data analysis were performed by J.J.Z and A.M.D., and K.R.R. All other experiments were

559 performed and analyzed by J.J.Z., G.S, E.A.K., and K.R.R. The manuscript was written by

560 J.J.Z., E.A.K, and K.R.R. with input from all authors.

\section{Declaration of interests}

563 The authors declare no competing interests.

\section{Figure Legends}

566 Figure 1. Suppressor mutations affecting fur or O-antigen biosynthesis permit the loss of $c t p A$.

567 (A) Strategy for isolation of $\triangle c t p A$ suppressor mutants. (B) Viability of suppressor strains during 568 CtpA depletion on PYED. (C) Differential interference contrast (DIC) images of the indicated 
strains grown in CtpA-expressing (PYEX) or non-expressing (PYED) conditions. D) DIC images of the indicated strains grown to exponential phase in PYE. Scale bar, $3 \mu \mathrm{m}$.

572 Figure 2. $\Delta c t p A$ and $\Delta / p x C$ strains with suppressor mutations contain little or no lipid $A$. (A)

573 Endotoxin units $(E U)$ per $\mathrm{mL}$ of whole cells of the strains indicated in panel $B$ (mean \pm S.D.,

$574 N=3$ ). Dots represent individual data points, and mean values are displayed above bars. (B) Hot

575 aqueous-phenol LPS extracts of the indicated strains. ${ }^{* *}=$ S-LPS, ${ }^{* *}=$ putative full-length lipid

576 A-core, * = putative lipid A. (C) Lipid A (left) or rough LPS (right) extracted from the indicated

577 strains. ${ }^{*}=$ lipid A. ${ }^{* *}=$ rough LPS. (D) Proteinase K-treated lysates of the indicated strains.

578 Cells were maintained in PYED or shifted into PYEX for 6 hours before harvesting. Samples

579 were normalized by $\mathrm{OD}_{660}$. NA1000 was grown in PYE. Leaky expression of LpxC can generate

580 S-LPS $\left({ }^{* *}\right)$ and lipid A + core $\left(^{* *}\right)$ in PYED. Full-length S-LPS is not restored to ctpA and the

581 lipid A-core species is reduced in size $\left({ }^{*}\right)$ because CCNA_03733 is needed for mannose

582 incorporation. (E) Electron cryotomography images of the indicated strains noting the inner

583 membrane (IM), peptidoglycan (PG), outer membrane (OM), and S-layer. All strains were grown

584 to exponential phase in PYE medium, except that CtpA was depleted from KR3906 during 12

585 hours of growth in PYED prior to analysis. Scale bars, $100 \mathrm{~nm}$.

587 Figure 3. Deletion of fur supports the viability of $\Delta / p x C$ cells. (A) DIC images of the LpxC

588 depletion strain alone or harboring the indicated mutations, grown in PYEX or PYED for 10

589 hours. Scale bar, $3 \mu \mathrm{m}$. (B) Viability of the LpxC depletion strain, alone or harboring the

590 indicated mutations, plated on PYEX or PYED. (C) Viability of $\Delta / p x C \Delta$ fur $\Delta s s p B$ cells harboring

591 a Pxyl-fur or a Pxyl-cerulean expression vector. Plates included kanamycin to retain expression

592 vectors.

594 Figure 4. Fur-regulated processes control the conditional essentiality of lipid A. (A) Sensitivity to 595 the indicated chemicals measured by disc diffusion assay. Dots indicate individual data points.

596 Suppressor mutations present in strains represented by blue or green bars are, from left to right, $597 \triangle C C N A \_00497, \triangle C C N A \_01553, \triangle C C N A \_03733$, and $\triangle$ fur. (B) CHIR-090 sensitivity measured

598 by disc diffusion assay. Partial clearing indicates the diameter of a ring of intermediate growth.

599 Dots and triangles indicate individual measurements of cleared and partially cleared zones, 600 respectively (mean \pm S.D., $N=3$ ). (C) Viability of the LpxC depletion strain in inducing (PYEX) or 601 depleting (PYED) conditions, in the presence or absence of $100 \mu \mathrm{M} 2,2$ '-dipyridyl. (D) Viability of 602 the $\Delta / p x C \Delta$ fur $\Delta s s p B$ strain harboring a Pxyl-fur plasmid, grown in noninducing (PYED) or 
603 inducing (PYEX) conditions, in the presence or absence of $100 \mu \mathrm{M} 2,2$ '-dipyridyl. Plates

604 included kanamycin to retain the expression vector. Brightness was reduced and contrast

605 increased to improve the clarity of colonies grown on 2,2'-dipyridyl. (E) Proteinase K-treated

606 lysates of the indicated strains grown overnight in the presence or absence of $100 \mu \mathrm{M} 2,2$ '-

607 dipyridyl. Samples were normalized by OD $_{660}$. (F) Growth inhibition by SNG in liquid PYE

608 cultures of the indicated strains. Dots represent individual $\mathrm{OD}_{660}$ ratios (mean \pm S.D.). (G)

609 Genes regulated by Fur in concert with iron (sets A and B) are modulated similarly by deletion of

610 fur or by iron limitation, while genes regulated by iron alone (sets $C$ and D) are modulated in

611 opposite directions. Changes in Fur-regulated gene expression correlate with the ability to lose

612 lipid A.

613

614 Figure 5. RB-Tnseq identifies sphingolipid synthesis genes needed for fitness when LpxC is 615 inhibited. (A) Average gene fitness scores for three challenges of the NA1000 RB-Tnseq library 616 with PYE or PYE $+2 \mu \mathrm{g} / \mathrm{ml}$ CHIR-090. Fitness scores are color-coded based on regulation of the 617 corresponding genes. Fitness scores of selected genes (not regulated by Fur or iron) are

618 indicated by colors matching the open reading frame diagram below. (B) CHIR-090 sensitivity of 619 the indicated strains measured by disc diffusion assay (mean \pm S.D.) Where indicated, $0.5 \mathrm{mM}$ 620 vanillate was included in the medium. (C) Overnight growth of strains expressing (xylose) or 621 depleting (dextrose) LpxC, and expressing (vanillate) or not expressing the indicated genes.

622 Dots represent individual measurements (mean \pm S.D.).

624 Figure 6. CCNA_01217-01219 convert neutral ceramide to an anionic sphingolipid, ceramide 625 phosphoglycerate. (A) Extracted ion-chromatograms identified the indicated sphingolipid 626 species in NA1000 lipid extracts. (B) Structural determination of anionic sphingolipids was

627 performed by MS/MS analysis. (C) The presence of the indicated sphingolipids was assessed in 628 each deletion mutant and its respective complemented strain. $x$, no lipid of that type was 629 detected in the indicated strain. (D) Proposed mechanism for CPG2 synthesis.

631 Figure 7. Ceramide phosphoglycerate mediates susceptibility to colistin. (A) Overnight growth 632 of the indicated strains in the presence or absence of $10 \mu \mathrm{g} / \mathrm{ml}$ colistin. Dots represent individual $633 \mathrm{OD}_{660}$ measurements (mean \pm S.D.). (B) Model of the Caulobacter cell envelope containing LPS 634 and CPG2. Consequences for OM composition and colistin sensitivity when either lipid $\mathrm{A}(\Delta / \mathrm{pxC}$ $635 \Delta$ fur) or sphingolipids $(\Delta s p t)$ are eliminated. The presence of CPG2 and the absence of fur are 636 together required for the viability of lipid A-deficient Caulobacter. 


\section{Methods}

641 Growth conditions. All Caulobacter crescentus strains were derived from NA1000 (Evinger

642 and Agabian, 1977) and are listed in Table S4. Unless otherwise stated, Caulobacter was

643 grown in peptone-yeast extract medium (PYE) (Ely, 1991) at $30^{\circ} \mathrm{C}$. Solid media were prepared

644 using Fisher agar (BP1423). PYE was supplemented with $0.3 \%$ xylose (PYEX) or 0.2\% D-

645 glucose (PYED) where indicated. When changing between inducing and non-inducing

646 conditions, cells were washed twice with PYE medium lacking additional sugars before being

647 released into or plated on medium supplemented with a different sugar. Counter-selection using

648 sacB was performed using $3 \%$ sucrose. $100 \mu \mathrm{M}$ 2,2'-dipyridyl was added to culture media to

649 achieve low-iron conditions. Vanillic acid was added to PYE media at a final concentration of 0.5

$650 \mathrm{mM}$ to drive gene expression from the vanA promoter. Antibiotics added to PYE were used at

651 the following concentrations $(\mu \mathrm{g} / \mathrm{mL})$ for liquid $(\mathrm{L})$ or solid $(\mathrm{S})$ medium: kanamycin, $5(\mathrm{~L}), 25(\mathrm{~S})$;

652 chloramphenicol, 1 (L/S); nalidixic acid, 20 (S); gentamycin, 25 (L), 5 (S); oxytetracycline, 1 (L),

653 (S); spectinomycin, 25 (L), 100 (S); hygromycin, 100 (L/S); streptonigrin 0.025 or 0.25 (L). E.

654 coli was grown in lysogeny broth $(10 \mathrm{~g} / \mathrm{L}$ tryptone, $5 \mathrm{~g} / \mathrm{L}$ yeast extract, $5 \mathrm{~g} / \mathrm{L} \mathrm{NaCl})$ at $37^{\circ} \mathrm{C}$,

655 supplemented with antibiotics at the following concentrations $(\mu \mathrm{g} / \mathrm{mL})$ for liquid $(\mathrm{L})$ or solid $(\mathrm{S})$

656 medium: kanamycin, 30 (L), 50 (S); chloramphenicol, 20 (L), 30 (S); gentamycin, 15 (L), 20 (S);

657 tetracycline, 12 (L/S); spectinomycin, 50 (L/S); hygromycin, 100 (L/S). Diaminopimelic acid (0.3

$658 \mathrm{mM}$ ) was added to solid or media to support the growth of E. coli strain WM3064 (Dehio and

659 Meyer, 1997).

660

661 Plasmid construction. Plasmid descriptions are listed in Table S5. Primer sequences used for

662 plasmid construction are listed in Table S6.

663

664 pZIK133. The LpxC depletion vector was constructed by placing the IpxC coding region, C-

665 terminally fused to a 3xFLAG tag (amino acid sequence: DYKDHDGDYKDHDIDYKDDDDK)

666 followed by the Caulobacter ssrA tag (amino acid sequence: AANDNFAEEFAVAA), under

667 control of the $x y I X$ promoter. The $x y I X$ promoter was amplified using the pJS14-PxylX and

668 PxylX-IpxC R primers. The PxylX-IpxC F and IpxC-3xFLAG R primers were used to amplify

669 IpxC. The C-terminal fusion was amplified from pAB6 using the IpxC-3xFLAG F and ssrA-pJS14 
670 primers. The final plasmid was assembled via Gibson cloning into a BamHI/EcoRI-digested

671 pJS14 backbone.

672

$673 p Z I K 134$. For the IpxC knockout construct, flanking homology regions were amplified using the 674 primers IpxC UpF and IpxC UpR for the 5'- region, and IpxC DownF and IpxC DownR for the 3'675 region. The 5'- arm included a 5'- Spel site and a 3'- EcoRI site, and the 3'- arm included a 5'-

676 EcoRI site and a 3'- Sphl site. These fragments were digested with the indicated enzymes and 677 ligated into Spel/Sphl-digested pNPTS138. This intermediate plasmid was linearized with 678 EcoRl, and the EcoRl-digested tetAR cassette from pKOC3 was inserted to make the final 679 construct. pZIK73 and pZIK78. For knockouts of CCNA_01553 or CCNA_00497, flanking homology 682 regions were amplified using the following primer pairs: pZIK73 5'- region (01553 UpF; 01553 683 UpR), pZIK73 3'- region (01553 DownF; 01553 DownR), pZIK78 5'- region (00497::hyg UpF; 684 00497::hyg UpR), pZIK78 3'- region (00497::hyg DownF; 00497::hyg DownR). For each 685 construct, the 5'- arm included a 5'- Spel site and a 3'- Smal site, and the 3'- arm included a 5'686 Smal site and a 3'- EcoRI site. These fragments were digested with the indicated enzymes and ligated into Spel/EcoRI-digested pNPTS138. The intermediate plasmids were linearized with

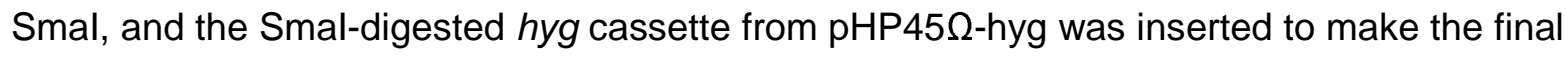
constructs.

pZIK80, pZIK81, pZIK82, and pZIK161. For the knockouts of CCNA_03733, CCNA_01068,

692 CCNA_01055, or CCNA_00055, flanking homology regions were amplified using the following 693 primer pairs: pZIK80 5'- region (03733::hyg UpF; 03733::hyg UpR), pZIK80 3'- region

694 (03733::hyg DownF; 03733::hyg DownR), pZIK81 5'- region (01068::hyg UpF; 01068::hyg UpR), 695 pZIK81 3'- region (01068::hyg DownF; 01068::hyg DownR), pZIK82 5'- region (01055::hyg UpF; 696 01055::hyg UpR), pZIK82 3'- region (01055::hyg DownF; 01055::hyg DownR), pZIK161 5'-

697 region (fur UpF; fur UpR), pZIK161 3'- region (fur DownF; fur DownR). Each 5'- arm included a 698 5'- Spel site and a 3'- BamHI site, and each 3'- arm included a 5'- BamHI site and a 3'- EcoRI 699 site. These fragments were digested with the indicated enzymes and ligated into Spel/EcoRI700 digested pNPTS138. The intermediate plasmids were linearized with BamHI, and the BamHI-

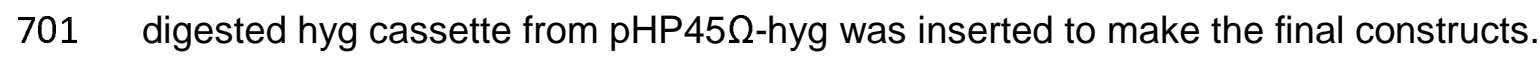


703

704

705

706

707

708

709

710

711

712

713

714

715

716

717

718

719

720

721

722

723

724

725

726

727

728

729

730

731

732

733

734

735 pKR432-4. For appending a C-terminal FLAG tag to the CCNA_01218-20 open reading frames.

736 The indicated genes were amplified from NA1000 genomic DNA using primer pairs Nde-

pZIK172-174. CCNA_00497, CCNA_01553, or CCNA_03733 were placed under control of the

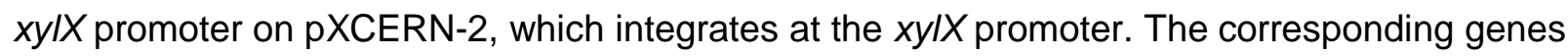
were initially cloned into pVCERN-2 before being moved into pXCERN-2. Genes were amplified with the following primer pairs: CCNA_00497 (pVCERN-2 00497 F; pVCERN-2 00497 R), CCNA_01553 (pVCERN-2 01553 F; pVCERN-2 01553 R), CCNA_03733 (pVCERN-2 03733 F; pVCERN-2 03733 R). Primer sets replace the start codon with an Ndel site and add a Sacl site after the stop codon. The corresponding gene fragment and pVCERN-2 were digested with Ndel and Sacl and ligated together. An Ndel/Mlul fragment was subsequently excised from each vector and moved to pXCERN-2 cut with the same enzymes.

pZIK175. CCNA_00055 (fur) was placed under control of the xyIX promoter on pXCERN-2, which integrates at the $x y I X$ promoter. CCNA_00055 was initially cloned into pVCERN-2 before being moved into pXCERN-2. CCNA_00055 was amplified using the Pvan-fur and fur-pVCERN primers, and this fragment was inserted into Ndel/Sacl-digested pVCERN-2 via Gibson assembly. The Ndel/Mlul fragment was subsequently excised and ligated into Ndel/Mluldigested pXCERN-2.

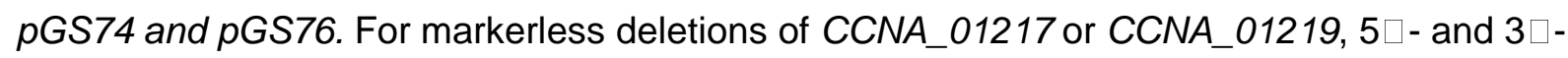

flanking homology regions, respectively, were amplified using the primer pairs EK1047/1048 and EK1049/1050 (CCNA_01217) and EK1055/1056 and EK1057/1058 (CCNA_01219). pNPTS138 was amplified with primers EK897/898, and vectors were constructed by Gibson assembly.

pKR429. For markerless deletion of CCNA_01218,5 $\square$ - and $3 \square$ - flanking homology regions, respectively, were amplified using the primer pairs 01218 up_fwd/01218 up_rev and 01218 down_fwd/01218 down_rev.pNPTS138 was digested with EcoRI and HindIII, and the vector was constructed by Gibson assembly.

pEK406. For complementing the deletion of CCNA_01217 in LC-MS/MS experiments, CCNA_01217-FLAG was amplified using primers EK1357/1358. The PCR product was ligated into the Ndel/Nhel sites of pVCHYC-5. 

using Ndel/Mlul and ligated into pFLGC-1 digested with the same enzymes.

pKR435. For expressing CCNA_01218-FLAG from the chromosomal vanA promoter.

741 CCNA_01218-FLAG was amplified from pKR432 using primers 01218-FLAG F/01218-FLAG R

742 and inserted in Ndel-digested pVGFPC-2 by Gibson assembly.

$744 p K R 436$. For expressing CCNA_01219-FLAG from the chromosomal vanA promoter.

745 CCNA_01219-FLAG was amplified from pKR433 using primers 01219-FLAG F/01219-FLAG R

746 and inserted in Ndel-digested pVGFPC-2 by Gibson assembly.

pKR437. For expressing CCNA_01220-FLAG from the chromosomal vanA promoter.

749 CCNA_01220-FLAG was amplified from pKR434 using primers 01220-FLAG F/01220-FLAG R 750 and inserted in Ndel-digested pVGFPC-2 by Gibson assembly.

pKR438. For expressing CCNA_01217-FLAG from the chromosomal vanA promoter. CCNA_01217-FLAG was amplified from pEK406 using primers 01217-FLAG F/01217-FLAG R and inserted in Ndel-digested pVGFPC-4 by Gibson assembly.

Strain construction. Unless otherwise stated, plasmids were mobilized from $E$. coli into $C$. crescentus by conjugation. E. coli donors were counterselected by the addition of nalidixic acid, or when WM3064 was used as the donor, by omitting diaminopimelic acid from selection plates. Gene deletion or disruption was achieved by two-step homologous recombination using sacB counterselection (Ely, 1991).

CtpA depletion strain. In the CtpA depletion strain (KR3906, Shapland et al., 2011), regulated depletion of CtpA is achieved by expressing $\operatorname{ctp} A:: 3 x F L A G:: s s r A$ from a xylose-inducible

764 promoter (Meisenzahl et al., 1997) on a high-copy plasmid in a $\Delta c t p A$ strain also lacking the 765 proteolytic adaptor sspB (Levchenko et al., 2000). The native CtpA protein could not be 766 depleted without an ssrA tag to target it for proteolysis. However, addition of this tag made CtpA 767 proteolysis so rapid that xylose-dependent expression of CtpA-3xFLAG-ssrA did not support

768 Caulobacter viability. Further deleting $s s p B$, which encodes a proteolytic adaptor for ssrA769 tagged substrates, reduced the basal rate of CtpA-3xFLAG-ssrA degradation enough to permit 770 complementation in PYE medium containing xylose (PYEX). 
772 LpxC depletion strain. The LpxC depletion strain KR4007 was constructed in a parallel manner

773 to the CtpA depletion strain KR3906. pZIK133 was introduced to KR1499 $(\Delta s s p B)$ by

774 conjugation and selection on PYE/chloramphenicol. pZIK134 was conjugated into this

775 intermediate strain, and colonies were selected on PYEX/chloramphenicol/oxytetracycline. After

776 sacB counterselection on PYEX/sucrose/oxytetracycline, colonies were screened for

777 chloramphenicol $^{\mathrm{R}}$ kanamycin $^{\mathrm{S}}$ on PYEX.

Stable $\triangle \operatorname{ctp} A$ or $\Delta l p x C$ strains. To generate stable $\Delta \operatorname{ctp} A$ or $\Delta / p x C$ strains without covering

780 plasmids, candidate suppressor genes identified by screening were disrupted in KR3906 or

781 KR4007, respectively, using two-step homologous recombination while cultivating the cells on

782 PYEX. Intermediate strains $\left(\right.$ sucrose $^{R}$ hygromycin $^{R}$ kanamycin $^{S}$ ) were grown in liquid PYED

783 without chloramphenicol to permit loss of the $\operatorname{ctp} A$ or $\operatorname{lp} x C$ covering plasmid, plated on PYED,

784 and tested for chloramphenicol ${ }^{S}$. Absence of $\operatorname{ctp} A$ was confirmed using primers $\operatorname{ctpA} \mathrm{KO} F$ and

$785 \operatorname{ctpA} K O \mathrm{R}$, and absence of $I p x C$ was confirmed using primers IpxC KO F and IpxC KO R. The genomes of $\Delta c t p A \Delta f u r \Delta s s p B(\mathrm{KR} 4102)$ and $\Delta l p x C \Delta$ fur $\Delta s s p B(\mathrm{KR} 4103)$ were resequenced and contained no additional mutations. Stable $\Delta \operatorname{ctp} A$ or $\Delta / p x C$ strains were further modified by electroporation with purified plasmids (Gilchrist and Smit, 1991) to restore xylose-driven suppressor gene expression.

Unmarked deletions in genes for ceramide phosphoglycerate synthesis. Deletions in

792 CCNA_01217, CCNA_01218, CCNA_01219, and CCNA_01220 in NA1000 or KR4077 were

793 made by conjugation of the appropriate pNPTS138-based plasmid, followed by selection on

794 PYE/kanamycin/nalidixic acid. After overnight growth in PYE, cells were plated on

795 PYEX/sucrose, and sucrose ${ }^{R}$ colonies were screened for kanamycin ${ }^{S}$. Colony PCR with the

796 following primers was used to detect the deletion of the indicated chromosomal genes:

797 CCNA_01217, EK S238/S239; CCNA_01218, EK S240/S241; CCNA_01219, EK S242/S243;

798 CCNA_01220, EK S216/S217. Loci were sequenced with the indicated primers to ensure the

799 accuracy of in-frame deletions. Unmarked deletions of CCNA_01217, CCNA_01218,

800 CCNA_01219, and CCNA_01220 were made in KR4091 by conjugation of KR4091 with

801 WM3064 harboring the appropriate pNPTS138-based plasmids, followed by selection on

802 PYEX/kanamycin medium omitting diaminopimelic acid. After growth overnight in PYEX, cells 803 were plated on PYEX/sucrose, and sucrose ${ }^{R}$ colonies were screened for kanamycins. Colony 804 PCR with the following primers was used to detect the deletion of the indicated chromosomal 

S242/S243; CCNA_01220, EK S216/S217. Loci were sequenced with the indicated primers to ensure the accuracy of in-frame deletions. Strains were screened for oxytetracycline ${ }^{R}$ and hygromycin $^{R}$ to ensure that they maintained deletions of $I p x C$ and fur, respectively.

809

Complementation of genes for ceramide phosphoglycerate synthesis. To complement deletions of CCNA_01217-01220, the following plasmids were introduced by conjugation to place the complementing gene under control of the chromosomal vanA promoter: $\triangle C C N A \_01217$,

813 pEK406 (for LC-MS studies) or pKR438 (for growth and chemical sensitivity assays);

$814 \triangle C C N A \_01218, p K R 435 ; \triangle C C N A \_01219$, pKR436; or $\triangle C C N A \_01220$, pKR437. When

815 introducing plasmids into strains capable of IpxC depletion (based on KR4091), plasmids were

816 delivered from WM3064 to avoid the use of multiple antibiotics for selection/counterselection.

817 Correct integration of plasmids at the vanA locus was confirmed by colony PCR using primers

818 RecUni-1 and RecVan-2 (Thanbichler et al., 2007).

Suppressor selection. KR3906 was grown to full density in PYEX. $300 \mu \mathrm{L}$ of culture was

821 transferred onto an open, sterile Petri dish and mutagenized in a UV Stratalinker 1800

822 (Stratagene) with $30,000 \mu \mathrm{J}$ of energy. Mutagenized cells were plated on PYED. Recovered

823 colonies were passaged in liquid PYED overnight to allow loss of the covering plasmid, and

824 samples were streaked onto PYED. Isolated colonies were screened for chloramphenicol

825 sensitivity. Chlor $^{S}$ isolates were grown in PYE and saved at $-80^{\circ} \mathrm{C}$ in $10 \%$ dimethylsulfoxide.

826 Loss of $\operatorname{ctp} A$ was confirmed via PCR using the primers ctpA KO F and ctpA KO R, which anneal

827 to the interior of the open reading frame.

Genome resequencing. Strains were grown to full density in PYE, and genomic DNA was

830 extracted using the Quick-DNA Miniprep Kit (Genesee) or the DNeasy Blood \& Tissue Kit

831 (Qiagen). Genomic DNA was submitted to the UC Berkeley Functional Genomics Laboratory,

832 where libraries were prepared using a PCR-free protocol with multiplexing

833 (http://qb3.berkeley.edu/gsl/). Samples were sequenced at the UC Berkeley Vincent J. Coates

834 Genomics Sequencing Laboratory using a 300PE or 150PE MiSeq v3 run. Genomic sequencing 835 data were analyzed for variants using the Galaxy platform at usegalaxy.org (Afgan et al., 2016).

836 Adapter sequences were removed using Cutadapt, and sequences were aligned to the NA1000 837 genome (Marks et al., 2010) using Bowtie2. FreeBayes was used to analyze the BAM files for 838 variants. Variants with quality scores below 300 were discarded as noise. 
Growth and viability assays. For plate assays, strains were grown to $\mathrm{OD}_{660}=0.2-0.5$ in

841 permissive media, washed twice in PYE medium with no additions, and diluted to $\mathrm{OD}_{660}=0.1$.

$84210 \mu \mathrm{l}$ drops of ten-fold serial dilutions were pipetted onto permissive and nonpermissive media.

843 Plates were incubated for 3 days at $30^{\circ} \mathrm{C}$, and images are representative of at least three

844 independent trials. For end-point growth assays in liquid media, strains were grown in

845 permissive media to $\mathrm{OD}_{660}=0.2-0.5$. After washing in PYE medium without additions, cells were

846 resuspended at $\mathrm{OD}_{660}=0.01$ in permissive and nonpermissive media. $\mathrm{OD}_{660}$ values were

847 measured after $24 \mathrm{~h}$ growth at $30^{\circ} \mathrm{C}$.

849 Disc diffusion assays of chemical sensitivity. Cultures were grown to mid-exponential phase

$850\left(\mathrm{OD}_{660}\right.$ 0.2-0.5), and an amount of cells equivalent to $250 \mu \mathrm{l}$ of culture at $O D_{660}=0.2$ was added

851 to $4 \mathrm{ml}$ of PYE swarm agar $\left(0.3 \% \mathrm{w} / \mathrm{v}\right.$ agar) pre-warmed to $42^{\circ} \mathrm{C}$. Swarm agar containing

852 bacteria was spread onto solid PYE and allowed to set. Antibiotics or detergents (10 $\mu$ l each)

853 were added to sterile Whatman filter disks and allowed to dry in a fume hood before discs were

854 placed onto swarm agar surfaces. Plates were incubated upright at $30^{\circ} \mathrm{C}$ for 24 hours. The

855 diameters of the zones of clearing or haze were measured, and the diameter of the disk $(6 \mathrm{~mm})$

856 was subtracted from all measurements to yield the reported values. The total amount of

857 antibiotic or detergent added to each disk is as follows: kanamycin (100 $\mu \mathrm{g})$, rifampicin $(100 \mu \mathrm{g})$,

858 vancomycin $(1 \mathrm{mg}), \mathrm{CHIR}-090(100 \mu \mathrm{g}, \mathrm{APExBIO})$, bacitracin $(50 \mu \mathrm{g})$, TWEEN $20(10 \mu \mathrm{L}$ of

$85910 \%$ solution), Triton X-100 (10 $\mu \mathrm{L}$ of $10 \%$ solution), sodium dodecyl sulfate ( $10 \mu \mathrm{L}$ of $10 \%$

860 solution). Tests using CHIR-090 used one quarter of the standard amount of cells to reduce

861 growth haze. For strains overexpressing genes integrated at the vanA locus, uninduced cells

862 were grown in PYE/kanamycin or PYE/gentamicin, and aliquots were plated in PYE swarm agar

863 on PYE medium. Induced cells were grown in PYE/kanamycin or PYE/gentamicin containing 0.5

$864 \mathrm{mM}$ vanillate before plating in/on PYE medium containing $0.5 \mathrm{mM}$ vanillate. 100 M 2,2'-

865 dipyridyl was included in media for testing chemical sensitivity in iron-restricted conditions.

867 Streptonigrin sensitivity. Isolated colonies of the indicated strains were grown in PYE medium

868 to $\mathrm{OD}_{660}=0.2-0.5$ and diluted to $\mathrm{OD}_{660}=0.01$. The diluted culture was aliquoted into separate

869 tubes, which received $0.025 \mu \mathrm{g} / \mathrm{ml}, 0.25 \mu \mathrm{g} / \mathrm{ml}$, or no streptonigrin (SNG). After $24 \mathrm{~h}$ of growth at

$87030^{\circ} \mathrm{C}, \mathrm{OD}_{660}$ values were measured, and optical density ratios $(0.25 \mu \mathrm{g} / \mathrm{ml} \mathrm{SNG/no} \mathrm{addition} \mathrm{and}$

$8710.025 \mu \mathrm{g} / \mathrm{ml} \mathrm{SNG/no}$ addition) were calculated as a measure of growth inhibition. 

Assay kit (GenScript) was used to determine endotoxin units $/ \mathrm{mL}$ of culture. Cells were grown to mid-exponential phase $\left(O D_{660} 0.2-0.5\right)$, washed twice with non-pyrogenic LAL reagent water, and normalized in this water to $\mathrm{OD}_{660}=0.1$. Cell suspensions were serially diluted in nonpyrogenic water and analyzed according to manufacturer's instructions. whole-cell lysates, cells were harvested after overnight growth in the indicated medium. All cultures were normalized by $\mathrm{OD}_{660}$, pelleted, and resuspended to $100 \mu \mathrm{L}$ in $1 \mathrm{x}$ tricine loading buffer (100 mM Tris-HCl pH 6.8, 1\% sodium dodecyl sulfate (SDS), 20\% glycerol, 0.02\% Coomassie G-250, 1\% 2-mercaptoethanol). Proteinase K (125 ng/ $\mu \mathrm{L}$ ) was added, and samples were incubated overnight at $55^{\circ} \mathrm{C}$. Lysates were boiled $5 \mathrm{~min}$, and equal volumes $(10 \%$ of each sample) were analyzed by gel electrophoresis.

Hot aqueous-phenol LPS extractions were adapted from Westpahl and Jann (Davis and Goldberg, 2012; Westphal and Jann, 1965). $1 \mathrm{~mL}$ of culture at $\mathrm{OD}_{660}=0.75$ was pelleted and resuspended in $200 \mu \mathrm{L} 1 \mathrm{x}$ tricine loading buffer. Suspensions were boiled for $15 \mathrm{~min}$ and cooled to room temperature. $5 \mu \mathrm{L}$ of $20 \mathrm{mg} / \mathrm{mL}$ Proteinase $\mathrm{K}$ (Thermo) was added to each sample before incubation at $55^{\circ} \mathrm{C}$ for three hours. Suspensions were mixed with $200 \mu \mathrm{L}$ ice-cold Trissaturated phenol, vortexed, and incubated at $65^{\circ} \mathrm{C}$ for 15 minutes before being cooled to room temperature. $1 \mathrm{~mL}$ diethyl ether was added to each sample before vortexing and spinning for 10 minutes in a table-top centrifuge at $16,000 \times \mathrm{g}$. The bottom blue layer was removed to a fresh tube, and the extraction was repeated on the blue layer starting from the phenol step. $200 \mu \mathrm{L} 2 \mathrm{x}$ tricine loading buffer was added to each sample before gel electrophoresis.

Rough LPS was extracted by the method of Darveau and Hancock (Darveau and Hancock, 1983), modified as described (Hershey et al., 2019), beginning with 50 ml PYE cultures grown to $\mathrm{OD}_{660}=0.85$. Cultures were centrifuged, and cell pellets were resuspended in $2 \mathrm{ml} 10 \mathrm{mM}$ Tris- $\mathrm{HCl}$ ( $\mathrm{pH}$ 8.0) containing $2 \mathrm{mM} \mathrm{MgCl}_{2}$. Samples were sonicated (Qsonica Q500) on ice for $5 \mathrm{~min}$ at $20 \%$ amplitude, in cycles of $10 \mathrm{sec}$ on/20 sec off so that fewer than $5 \%$ of 901 cells remained intact. DNase I and RNase A were added to final concentrations of $100 \mu \mathrm{g} / \mathrm{ml}$ 902 and $25 \mu \mathrm{g} / \mathrm{ml}$, respectively, and lysates were incubated at $37^{\circ} \mathrm{C}$ for 1 hour. Additional DNase I 903 and RNase A were added to reach final concentrations of $200 \mu \mathrm{g} / \mathrm{ml}$ and $50 \mu \mathrm{g} / \mathrm{ml}$, respectively, 904 and lysates were incubated for 1 hour at $37^{\circ} \mathrm{C}$. SDS and EDTA were added to achieve final 905 concentrations of $2 \%$ and $100 \mathrm{mM}$, respectively, and lysates were incubated for $2 \mathrm{~h}$ at $37^{\circ} \mathrm{C}$ 906 before centrifugation (30 $\mathrm{min}$ at $\left.50,000 \times \mathrm{g}, 30 \mathrm{~min}, 4^{\circ} \mathrm{C}\right)$. Proteinase $\mathrm{K}(50 \mu \mathrm{g} / \mathrm{ml}$ ) was added to 
each supernatant, followed by incubation for $2 \mathrm{~h}$ at $60^{\circ} \mathrm{C}$. LPS was precipitated by the addition of 2 volumes of ice-cold $0.375 \mathrm{M} \mathrm{MgCl}_{2} / 95 \%$ ethanol and collected by centrifugation $(12,000 \times \mathrm{g}$, $\left.15 \mathrm{~min}, 4^{\circ} \mathrm{C}\right)$. Precipitates were resuspended in $3.3 \mathrm{ml} 10 \mathrm{mM}$ Tris- $\mathrm{HCl}(\mathrm{pH} 8.0) / 2 \% \mathrm{SDS} / 100$ $\mathrm{mM}$ EDTA and incubated with shaking overnight at $37^{\circ} \mathrm{C}$. Rough LPS was reprecipitated using 2 volumes ice-cold $0.375 \mathrm{M} \mathrm{MgCl}_{2} / 95 \%$ ethanol and collected by centrifugation $(12,000 \times \mathrm{g}, 15$

$\left.912 \min , 4^{\circ} \mathrm{C}\right)$. Precipitates were suspended in $10 \mathrm{mM}$ Tris- $\mathrm{HCl}(\mathrm{pH} 8.0)$ and centrifuged $(200,000 \mathrm{x}$

$\left.913 g, 2 \mathrm{~h}, 4^{\circ} \mathrm{C}\right)$. After removal of the supernatant by pipetting, LPS pellets were resuspended in 1

$914 \mathrm{mll} 1 \mathrm{x}$ tricine loading buffer, and $5 \mu \mathrm{l}$ were analyzed by gel electrophoresis.

$915 \quad$ Free lipid A was extracted by the Caroff method (El Hamidi et al., 2005), modified as

916 described (Leung et al., 2017), starting with $10 \mathrm{ml}$ of PYE culture grown to $O D_{600}=0.6$. Cultures

917 were divided into multiple tubes and centrifuged at $14,000 \times \mathrm{g}$ for $2 \mathrm{~min}$. In a fume hood, cell

918 pellets from each culture were resuspended, combined, and transferred to a gasketed

919 microcentrifuge tube using $250 \mu \mathrm{l} 70 \%(\mathrm{v} / \mathrm{v})$ isobutyric acid + $150 \mu \mathrm{l} 1 \mathrm{M}$ ammonium hydroxide.

920 Samples were incubated in a boiling water bath in a fume hood for $1 \mathrm{~h}$, with vortexing every 15

$921 \mathrm{~min}$. Samples were cooled on ice and centrifuged at $2000 \times \mathrm{g}$ for $15 \mathrm{~min}$. In a fume hood,

922 supernatants $(\sim 400 \mu \mathrm{l})$ were transferred to new gasketed tubes, each containing $400 \mu \mathrm{l}$

923 endotoxin-free water. Small holes were punched in the gasketed caps using a syringe needle

924 before the samples were frozen in liquid nitrogen and lyophilized overnight. Methanol (1 ml) was

925 added, and samples were sonicated in a water bath for $5 \mathrm{~min}$. Samples were centrifuged at

$92610,000 \times g$ for $5 \mathrm{~min}$, and methanol was aspirated. The methanol wash was repeated before

927 lipids were solubilized in $190 \mu$ 3:1.5:0.25 v/v/v chloroform:methanol:endotoxin-free water. After

928 vortexing, samples were centrifuged at 8,000 $\mathrm{g}$ for $5 \mathrm{~min}$. Supernatants were transferred to

929 fresh gasketed tubes, and extracts were dried under a stream of nitrogen before analysis by

930 mass spectrometry (see below) or gel electrophoresis. Samples for gel electrophoresis were

931 resuspended using $100 \mu \mathrm{l}$ x tricine loading buffer, and $10 \mu \mathrm{l}$ of each sample was analyzed.

932 All lipid samples were analyzed on 16.5\% Mini-PROTEAN Tris-Tricine gels (Bio-Rad).

933 Carbohydrates were stained using Pro-Q Emerald 300 Lipopolysaccharide Gel Stain Kit

934 (Molecular Probes; P20495) per manufacturer's instructions. For Western blot analysis of S-

935 LPS, equal numbers of cells grown in PYE with appropriate additions were pelleted,

936 resuspended in 1x SDS loading buffer, and boiled before analysis on $12 \%$ polyacrylamide gels

937 and transfer to Immobilon-P PVDF membranes. Blots were probed with a-S-LPS $(1: 20,000)$

938 (Walker et al., 1994) and horseradish peroxidase-conjugated anti-rabbit antibodies (1:5000) and

939 analyzed using Western Lightning. Stained lipid species were visualized using a Bio-Rad Gel

940 Doc XR. 
941 High performance liquid chromatography-tandem mass spectrometry (HPLC-MSMS) of

942 lipid A extracts. All samples for HPLC-electrospray ionization tandem mass spectrometry were

943 generated by the modified Caroff extraction protocol described above. Each extract was initially

944 dissolved in $100 \mu \mathrm{L} \mathrm{1:2} \mathrm{chloroform:} \mathrm{methanol} \mathrm{before} \mathrm{dilution} \mathrm{1:10} \mathrm{with} \mathrm{methanol} \mathrm{for} \mathrm{analysis.} \mathrm{A}$

$9452-5 \mu \mathrm{L}$ aliquot of each solution was injected onto a Phenomenex Jupiter C4 column $(2 \times 50 \mathrm{~mm}$,

$9465 \mu \mathrm{m}, 300 \AA$ A ) for HPLC-MSMS analysis with a Waters Acquity UPLC system coupled to a

947 Thermo LTQ-Orbitrap Velos Pro mass spectrometer, which was equipped with an atmospheric

948 pressure electrospray ionization source. For lipid detection, the HPLC-MSMS analyses were

949 carried out with full-mass detection over a mass range of $m / z 250$ to 2000 in the Fourier

950 transform MS mode, with negative-ion detection. The mass resolution was 60,000 FWHM @

$951 \mathrm{~m} / \mathrm{z}$ 400. Fragmentation product ion masses of the three most intense precursor ions were

952 measured in the ion trap or orbitrap (7500 resolution) mass analyzer using stepped collision-

953 induced dissociation (35\% of the normalized collision energy) or Higher energy collision-induced

954 dissociation (35\% of the normalized collision energy) activation energies. During data

955 acquisitions, real-time mass calibration was applied with $m / z 283.26454$ as the lock mass for

956 negative-ion detection. The mobile phase for separation was (A) $1 \mathrm{mM}$ ammonium acetate

957 solution and (B) 90\% (1:1 acetonitrile/propanol)/10\% water/1 mM ammonium acetate as the

958 binary solvents for the 16-minute gradient elution: 0 to $10 \mathrm{~min}, 30 \%$ to $100 \% \mathrm{~B}$; 10 to $12 \mathrm{~min}$,

$959100 \%$ B and 12 to $12.1 \mathrm{~min}$ at $30 \% \mathrm{~B}$, followed by column equilibration at 30\% B from 12.1 to 16

$960 \mathrm{~min}$. The column flow rate was $0.35 \mathrm{~mL} / \mathrm{min}$ and the column temperature was maintained at

$96140^{\circ} \mathrm{C}$.

962

963 Lipid A structure analysis. MALDI-TOF MS was used to screen lipid extracts. To check

964 structures, tandem MS and ancillary separation techniques were required. These are described

965 below. HPLC-MSMS (above) describes the generation of data for structure determinations in

966 Fig. S3. Notably, the triple deletion strain $\Delta c t p A \Delta$ fur $\Delta s s p B$ contained no lipid A with sugars at

967 the terminal ( 1 and $4^{\prime}$ ) positions but rather contained phosphates, as found in canonical lipid $A$

968 structures. The $\Delta / p x C \Delta f u r \Delta s s p B$ strain contained an ion at $1412 \mathrm{~m} / \mathrm{z}$, the structure of which

969 remains unclear. The HPLC-MSMS data of this ion showed no loss of phosphate, as seen in

$970 \Delta \operatorname{ctp} A \Delta$ fur $\Delta s s p B$, nor loss of sugars, as seen for the NA1000, $\Delta s s p B$, or $\Delta f u r \Delta s s p B$ strains.

971 The fragmentation pattern strongly suggested that something other than lipid $A$ was responsible

972 for the ion at $1412 \mathrm{~m} / \mathrm{z}$. Given that cardiolipin is a common microbial membrane lipid, we carried

973 out HILIC-MS (described below) with cardiolipin and lipid A standards. Both standards were

974 retained by HILIC, as expected for hydrophobic molecules, but extracts from the $\Delta / p x C \Delta$ fur 
$975 \triangle s s p B$ mutant showed no ions at all, suggesting that the species at $1412 \mathrm{~m} / \mathrm{z}$ is not hydrophobic

976 enough to be retained. Regrettably, there remains no structure identified for the ion at $1412 \mathrm{~m} / \mathrm{z}$.

977 Generally, structure analysis was conducted manually according to our prior effort in this field

978 (Yoon et al., 2016).

979

980 Hydrophobic interaction liquid chromatography-mass spectrometry (HILIC-MS). A 10- $\mu \mathrm{L}$

981 aliquot of each solution was injected into a Waters Atlantis HILIC column (4.6 mm x $150 \mathrm{~mm}, 5$

$982 \mu \mathrm{m})$ to run LC-MS on a Water Acquity UPLC system coupled to a Thermo LTQ-Orbitrap Velos

983 Pro mass spectrometer, which was equipped with an atmospheric pressure electrospray

984 ionization source. For lipid detection, the HILIC-MS runs were carried out with full-mass

985 detection over a mass range of $m / z 80$ to 2000 in the Fourier transform MS mode, with positive-

986 ion and negative-ion detection, respectively, in two rounds of LC injections. The mass resolution

987 was 60,000 FWHM @ m/z 400. During data acquisitions, real-time mass calibration was applied

988 with $\mathrm{m} / \mathrm{z} 391.28426$ as the lock mass for positive-ion detection and with $\mathrm{m} / \mathrm{z} 112.98563$ as the

989 lock mass for negative-ion detection. The mobile phase of HILIC was (A) $20 \mathrm{mM}$ ammonium

990 acetate solution ( $\mathrm{pH}$ adjusted to 4.0 with acetic acid) and (B) methanol as the binary solvents for

991 gradient elution: 0-4 min, $99 \%$ B; 4 to $12.5 \mathrm{~min}, 99 \%$ to $20 \%$ B and 12.5 to $15 \mathrm{~min}$ at $20 \% \mathrm{~B}$,

992 followed by column equilibration at $99 \%$ B for $5 \mathrm{~min}$ between injections. The column flow rate

993 was $0.4 \mathrm{~mL} / \mathrm{min}$ and the column temperature was maintained at $40^{\circ} \mathrm{C}$.

Differential interference contrast microscopy. Cells were immobilized on agarose pads (1\% $w / v$ in reverse osmosis-purified water). Images were taken using a Zeiss EC Plan-Neofluar

997 100x/1.3 Oil M27 objective on a Zeiss Axiolmager M1 microscope with a Hamamatsu Digital 998 CCD Camera (C8484-03G01). Images were acquired using iVision software and processed 999 using ImageJ.

1000

1001

CryoEM imaging and tomographic processing. Cultures $(5 \mathrm{~mL})$ of KR4000, KR4102,

$1002 \mathrm{KR} 4103$, and KR3906 grown to $\mathrm{OD}_{660}$ 0.2-0.5 were centrifuged $\left(4^{\circ} \mathrm{C}, 16,000 \times \mathrm{g}, 15\right.$ minutes),

1003 and cell pellets were resuspended in $50 \mu \mathrm{L}$ PYE. For KR3906, cells grown in PYEX were

1004 washed twice with PYE, released into PYED at $O_{660}=0.02$, and incubated for 12 hours before

1005 harvest. $3 \mu \mathrm{L}$ of cell suspension, mixed 1:1 with Fiducial markers (10-nm gold particles

1006 conjugated to Protein A; Aurion) was applied to glow-discharged quantifoil grids (R2/2) and

1007 frozen in liquid ethane using an automatic plunge freezing device (Vitrobot, $\mathrm{FEI} .12^{\circ} \mathrm{C}, 8-12 \mathrm{~s}$

1008 blot time, blot force 8 , humidity $100 \%$ ). 

with in column omega energy filter and K2 direct electron camera. Grids of KR4102 and KR3906 were imaged on a Krios Cryo TEM (FEI) operating at 300kV with post column energy

1012 filter (Quantum, GATAN) and K2 direct electron camera. All data were collected with the 1013 automatic data collection program serialEM (Mastonarde, 2005). Square overview images were 1014 acquired using a defocus of 80-100 microns at a nominal magnification of 3600-6500x (Krios) or

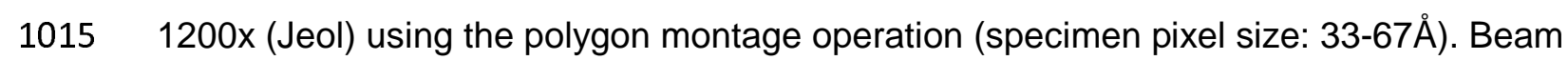

1016 intensity was set to $8 e^{-/ p x} / \mathrm{s}$ over an empty hole and exposure times ranged from $2-5 \mathrm{~s}$

1017 depending on ice thickness. Bidirectional tomographic tilt series were collected from $\pm 60^{\circ}$ using 1018 a defocus of 6-8 $\mu \mathrm{m}$ and at a magnification which provided specimen pixel size of $4-7 \AA$. Total 1019 dose of the tilt series were kept between 60-90 e $/ \AA^{2}$. All tilt series images were collected in 1020 movie mode and the frames aligned using MotionCor2 (Zheng et al., 2017). Aligned frames 1021 were compiled into stacks and processed using IMOD (Kremer et al., 1996). Contrast of 1022 resulting tomograms was enhanced using a non-linear anisotropic diffusion filter (Frangakis and 1023 Hegerl, 2001) and manually segmented using the 3D visualization program AMIRA

1024 (ThermoFisher).

RB-Tnseq analysis. A $1 \mathrm{ml}$ aliquot of the RB-Tnseq library in NA1000 (Price et al., 2018) was thawed and grown to $\mathrm{OD}_{660}=0.65 \mathrm{in} 25 \mathrm{ml}$ PYE medium with kanamycin. Aliquots of this culture were saved for sequencing of pre-challenge barcodes, or were diluted to $O D_{660}=0.02$ in PYE medium (set8IT011, set8IT023, and set8IT035) or PYE medium with $2 \mu \mathrm{g} / \mathrm{ml}$ CHIR-090 (set8IT012, set8IT024, and set8IT036). Cultures were grown for 9 hours at $30^{\circ} \mathrm{C}$ as described (Price et al., 2018) before cells were harvested and post-challenge barcodes were sequenced. Gene fitness (f) and significance (t) scores were calculated as described (Wetmore et al., 2015). Candidate genes examined in this study (CCNA_01217-01220) had fitness scores between -1.5 and -3.7 , with signficance scores between -3.0 and -8.4 , for individual trials of library growth in PYE + CHIR-090.

\section{Extraction and liquid chromatography-tandem mass spectrometry (LC-MS/MS) of}

1038 sphingolipids. Caulobacter strains were grown overnight with or without $0.5 \mathrm{mM}$ vanillate (5 $1039 \mathrm{ml}$ ), and lipids were extracted by the method of Bligh and Dyer (Bligh and Dyer, 1959). Cells 1040 were harvested and resuspended in $1 \mathrm{ml}$ of water, $3.75 \mathrm{ml}$ of $1: 2(\mathrm{v} / \mathrm{v})$ chloroform: methanol was 1041 added, and the samples were mixed by vortexing. Chloroform $(1.25 \mathrm{ml})$ and water $(1.25 \mathrm{ml})$ 1042 were added sequentially with vortexing to create a two-phase system and the samples were 
centrifuged at $200 \times g$ for 5 minutes at room temperature. The bottom, organic phase was

1044 transferred to a clean glass tube with a Pasteur pipette and washed twice in "authentic" upper

1045 phase. Subsequently, the organic phase containing lipids was collected and dried under argon.

1046 Our methods for lipid analysis by normal phase LC/ESI-MS/MS have been described (Guan et

1047 al., 2014). Briefly, normal phase LC was performed on an Agilent 1200 Quaternary LC system

1048 equipped with an Ascentis Silica HPLC column, $5 \mu \mathrm{m}, 25 \mathrm{~cm} \times 2.1 \mathrm{~mm}$ (Sigma-Aldrich, St.

1049 Louis, MO) as described. The LC eluent (with a total flow rate of $300 \mu \mathrm{l} / \mathrm{min}$ ) was introduced into

1050 the ESI source of a high resolution TripleTOF5600 mass spectrometer (Applied Biosystems,

1051 Foster City, CA). Instrumental settings for negative ion ESI and MS/MS analysis of lipid species

1052 were as follows: ion spray voltage (IS) $=-4500 \mathrm{~V}$; curtain gas (CUR) $=20$ psi; ion source gas 1

$1053(\mathrm{GSI})=20 \mathrm{psi}$; declustering potential $(\mathrm{DP})=-55 \mathrm{~V}$; and focusing potential $(\mathrm{FP})=-150 \mathrm{~V}$. The

1054 MS/MS analysis used nitrogen as the collision gas. Data analysis was performed using Analyst

1055 TF1.5 software (Applied Biosystems, Foster City, CA).

Data Availability Statement

1058 Sequence data that support the findings of this study are openly available in the

1059 Sequence Read Archive at https://www.ncbi.nlm.nih.gov/sra, under BioProject ID

1060 PRJNA526705, with specific NCBI BioSample accession numbers listed in Table S3. RB-Tnseq

1061 data are accessible at https://fit.genomics.lbl.gov/, with set and index numbers listed under RB-

1062 Tnseq analysis.

1063

\section{Supplemental Information Titles and Legends}

1065 Figure S1, related to Fig. 1: Morphology, growth, and complementation of strains in which

1066 suppressor mutations permit the loss of $c t p A$. (A) Isolates from the $\triangle c t p A$ suppressor screen

1067 show variations in morphology. DIC images of selected suppressor isolates confirmed to have

1068 lost the ctpA covering plasmid. Putative suppressor mutations identified by whole-genome

1069 resequencing are indicated. Scale bar, $3 \mu \mathrm{m}$. (B) Growth curves of the indicated strains in PYE 
showing (i or iii) $\mathrm{OD}_{660}$ and (ii or iv) colony-forming units (CFU) per $\mathrm{mL}$ (mean \pm S.D., $N=3$ ). (C) Viability assays of $\triangle \operatorname{ctp} A$ suppressor mutants, each harboring a vector for xylose-driven expression of the corresponding suppressor gene or the cerulean gene as a control. Kanamycin

1073 was included in media to retain expression vectors.

Figure S2, related to Fig.1: A subset of $\operatorname{ctpA}$ suppressor mutations impair or block S-LPS production. (A) a-S-LPS-probed immunoblot and (B) Pro-Q Emerald 300-stained gel of Proteinase K-treated whole-cell lysates of the indicated strains. (C) Complementation of Oantigen biosynthesis using plasmid-borne genes driven by a xylose-inducible promoter. Pro- $Q$ Emerald 300-stained polyacrylamide gel of Proteinase K-treated whole-cell lysates of the indicated strains grown in either PYED (D) or PYEX (X). Samples were normalized by $\mathrm{OD}_{660 .}{ }^{* * *}$ $=$ S-LPS, ${ }^{* *}=$ putative full-length lipid A-core polysaccharide, ${ }^{*}=$ putative incomplete lipid Acore species in cells lacking manC activity (CCNA_03733).

Figure S3, related to Figure 2: $\triangle \operatorname{ctp} A$ and $\Delta / p x C$ strains with suppressor mutations lack wildtype lipid A. Tandem mass spectrometry (MSMS)-derived structures of lipid A from the indicated strains. Lipid extraction and MSMS analysis were performed using the same protocols for all strains.

Movie S1, related to Figure 2. Tomogram of Caulobacter crescentus NA1000 grown to midexponential phase in PYE.

Movie S2, related to Figure 2. Tomogram of $\Delta c t p A \Delta s s p B \Delta$ fur grown to mid-exponential phase in PYE.

Movie S3, related to Figure 2. Tomogram of $\Delta l p x C \Delta s s p B \Delta f u r$ grown to mid-exponential phase in PYE.

Movie S4, related to Figure 2. Tomogram of $\triangle \operatorname{ctp} A \Delta s s p B+\operatorname{pJS} 14-\mathrm{P}_{x y \mid x}-\operatorname{ctp} A-3 x F L A G-s s r A$ grown to mid-exponential phase in PYEX, washed twice with PYE, released into PYED at $O_{660}$ $=0.02$, and incubated for 10 hours before harvest.

Figure S4, related to Figure 6: The total ion chromatogram of the C. crescentus lipidome shows the major lipid species present. DAG: diacylglycerol; MHDAG: mono-hexosyl 
1104 diacylglycerol; FA: fatty acids; PG: phosphatidylglycerol; HexA-DAG: hexuronic acid-

1105 diacylglycerol; CPG: ceramide phosphoglycerate.

Figure S5, related to Figure 3. Slow growth at a reduced temperature in rich medium is insufficient for viability of $\triangle / p x C$ strains. $(A)$ Viability of the LpxC depletion strain grown in inducing (PYEX) or depleting (PYED) conditions, at the indicated temperatures. $(B)$ Viability of the stable $\Delta / p x C \Delta$ fur $\Delta s s p B$ strain harboring a $P x y l$-fur plasmid, grown in noninducing (PYED) or inducing (PYED) conditions, at the indicated temperatures. Cells were plated from 10-fold serially diluted suspensions normalized to $\mathrm{OD}_{660}=0.1$. Plates were incubated for 3 days $\left(30^{\circ} \mathrm{C}\right)$ or 6 days $\left(22^{\circ} \mathrm{C}\right)$ and are representative of at least three independent trials. Plates in $B$ included kanamycin to retain the fur expression vector.

Table S1, related to Figure 1: Single-nucleotide polymorphisms and indels in $\triangle \operatorname{ctp} A$ suppressors.

Table S2, related to Figure 3: Single-nucleotide polymorphisms and indels in $\Delta / p x C$ suppressors.

Table S3, related to Figure 3: Growth rates in PYE medium at $22^{\circ} \mathrm{C}$.

Table S4, related to STAR Methods: Strains used in this study.

Table S5, related to STAR Methods: Plasmids used in this study.

Table S6, related to STAR Methods: Primers used in this study.

1131 Afgan, E., Baker, D., Beek, M. van den, Blankenberg, D., Bouvier, D., Cech, M., Chilton, J., 1132 Clements, D., Coraor, N., Eberhard, C., et al. (2016). The Galaxy platform for accessible, 1133 reproducible and collaborative biomedical analyses: 2016 update. Nucleic Acids Res. 44, W31134 W10. 
1138

Awram, P., and Smit, J. (2001). Identification of lipopolysaccharide $O$ antigen synthesis genes required for attachment of the S-layer of Caulobacter crescentus. Microbiology 147, 1451-1460.

Bligh, E.G., and Dyer, W.J. (1959). A rapid method of total lipid extraction and purification. Can J. Biochem. Physiol. 37, 911-917.

Blondelet-Rouault, M.H., Weiser, J., Lebrihi, A., Branny, P., and Pernodet, J.L. (1997). Antibiotic resistance gene cassettes derived from the omega interposon for use in $\mathrm{E}$. coli and Streptomyces. Gene 190, 315-317.

Boll, J.M., Crofts, A.A., Peters, K., Cattior, V., Vollmer, W., Davies, B.W., and Trent, M.S. (2016). A penicillin-binding protein inhibits selection of colistin-resistant, lipooligosaccharidedeficient Acinetobacter baumannii. Proc. Natl. Acad. Sci. USA 113, E6228-E6237.

Brown, D.B., Forsberg, L.S., Kannenberg, E.L., and Carlson, R.W. (2012). Characterization of galacturonosyl transferase genes rgtA, rgtB, rgtC, rgtD, and rgtE responsible for lipopolysaccharide synthesis in nitrogen-fixing endosymbiont Rhizobium leguminosarum: lipopolysaccharide core and lipid galacturonosyl residues confer membrane stability. J. Biol. Chem. 287, 935-949.

Brown, D.B., Muszynski, A., and Carlson, R.W. (2013). Elucidation of a novel lipid A alpha-(1,1)GalA transferase gene (rgtF) from Mesorhizobium loti: Heterologous expression of rgtF causes Rhizobium etli to synthesize lipid A with alpha-(1,1)-GalA. Glycobiology 23, 546-558.

Christen, B., Abeliuk, E., Collier, J.M., Kalogeraki, V.S., Passarelli, B., Coller, J.A., Fero, M.J., McAdams, H.H., and Shapiro, L. (2011). The essential genome of a bacterium. Mol. Syst. Biol. $7,528$.

Crosson, S., McGrath, P.T., Stephens, C., McAdams, H.H., and Shapiro, L. (2005). Conserved modular design of an oxygen sensory/signaling network with species-specific output. Proc. Natl. Acad. Sci. USA 102, 8018-8023.

\section{Darveau, R.P., and Hancock, R.E. (1983). Procedure for isolation of bacterial} lipopolysaccharides from both smooth and rough Pseudomonas aeruginosa and Salmonella typhimurium strains. J. Bacteriol. 155, 831-838. 
Davis, M.R.J., and Goldberg, J.B. (2012). Purification and visualization of lipopolysaccharide from Gram-negative bacteria by hot aqueous-phenol extraction. J. Vis. Exp. e3916.

De Castro, C., Molinaro, A., Lanzetta, R., Silipo, A., and Parrilli, M. (2008). Lipopolysaccharide structures from Agrobacterium and Rhizobiaceae species. Carbohydr. Res. 343, 1924-1933. Dehio, C., and Meyer, M. (1997). Maintenance of broad-host-range incompatibility group P and group Q plasmids and transposition of Tn5 in Bartonella henselae following conjugal plasmid transfer from Escherichia coli. J. Bacteriol. 179, 538-540. bacterial lipid $A$ : Easy and rapid method for mass spectrometric characterization. J. Lipid Res. 46, 1773-1778.

Ely, B. (1991). Genetics of Caulobacter crescentus. Methods Enzymol. 204, 372-384. crescentus stalked and swarmer cells. J. Bacteriol. 132, 294-301. regulator (Fur) reversibly binds a [2Fe-2S] cluster to sense intracellular iron homeostasis in Escherichia coli. J. Biol. Chem. 295, 15454-15463.

Frangakis, A.S., and Hegerl, R. (2001). Noise reduction in electron tomographic reconstructions using nonlinear anisotropic diffusion. J. Struct. Biol. 135, 239-250.

Gilchrist, A., and Smit, J. (1991). Transformation of freshwater and marine caulobacters by electroporation. J. Bacteriol. 173, 921-925. plasmalogen species of phospholipids and glycolipids. Biochim. Biophys. Acta 1842, 13531359. 
1210 Henry, R., Crane, B., Powell, D., Lucas, D.D., Li, Z., Aranda, J., Harrison, P., Nation, R.L.,

1211 Adler, B., Harper, M., et al. (2015). The transcriptomic response of Acinetobacter baumannii to

1212 colistin and diripenem alone and in combination in an in vitro

1213 pharmacokinetics/pharmacodynamics model. J. Antimicrob. Chermother. 70, 1303-1313.

Hershey, D.M., Fiebig, A., and Crosson, S. (2019). A genome-wide analysis of adhesion in Caulobacter crescentus identifies new regulatory and biosynthetic components for holdfast assembly. mBio 10, 2273.

Jones, M.D., Vinogradov, E., Nomellini, J.F., and Smit, J. (2015). The core and Opolysaccharide structure of the Caulobacter crescentus lipopolysaccharide. Carbohydr. Res. $402,111-117$.

Justino, M.C., Almeida, C.C., Teixeira, M., and Saraiva, L.M. (2007). Escherichia coli di-iron YtfE protein is necessary for the repair of stress-damaged iron-sulfur clusters. J. Biol. Chem. 282, 10352-10359. Chemical structure of glycosphingolipids isolated from Sphingomonas paucimobilis. FEBS Lett. 292, 107-110.

1236 The cell envelope structure of the lipopolysaccharide-lacking Gram-negative bacterium

1237 Sphingomonas paucimobilis. J. Bacteriol. 176, 284-290. dimensional image data using IMOD. J. Struct. Biol. 116, 71-76. 
Leung, L.M., Fondrie, W.E., Doi, Y., Johnson, J.K., Strickland, D.K., Ernst, R.K., and Goodlett, D.R. (2017). Identification of the ESKAPE pathogens by mass spectrometric analysis of microbial membrane glycolypids. Sci. Rep. 7, 6403. Levchenko, I., Seidel, M., Sauer, R.T., and Baker, T.A. (2000). A specificity-enhancing factor for the ClpXP degradation machine. Science 289, 2354-2356. Iron Content Is Critically Linked to the Fur Physiology in Shewanella oneidensis. Front.

1255 Microbiol. 11, 593246.

Mamat, U., Meredith, T.C., Aggarwal, P., Kuhl, A., Kirchoff, P., Lindner, B., Hanuszkiewicz, A., 1258 Sun, J., Holst, O., and Woodard, R.W. (2008). Single amino acid substitutions in either YhjD or 1259 MsbA confer viability to 3-deoxy-D-manno-oct-2-ulosonic acid-depleted Escherichia coli. Mol. Microbiol. 67, 633-648.

Marks, M.E., Castro-Rojas, C., Teiling, C., Du, L., Kapatral, V., Walunas, T.L., and Crosson, S. (2010). The genetic basis of laboratory adaptation in Caulobacter crescentus. J. Bacteriol. 192, 3678-3688.

Mastonarde, D.N. (2005). Automated electron microscope tomography using robust prediction of specimen movements. J. Struct. Biol. 152, 36-51. C.R. (2005). A slow, tight-binding inhibitor of the zinc-dependent deacetylase LpxC of lipid A biosynthesis with antibiotic activity comparable to ciprofloxacin. Biochemistry 44, 16574-16583. dependent promoter from Caulobacter crescentus. J. Bacteriol. 179, 592-600. the requisite lipopolysaccharide structure in Escherichia coli. ACS Chem. Biol. 1, 33-42. 
1281 is mediated by complete loss of lipopolysaccharide production. Antimicrob. Agents Chemother. $128254,4971-4977$.

Moffatt, J.H., Harper, M., and Boyce, J.D. (2019). Mechanisms of polymyxin resistance. Adv. Exp. Med. Biol. 55-71. Nachin, L., El Hassouni, M., Loiseau, L., Expert, D., and Barras, F. (2001). SoxR-dependent response to oxidative stress and virulence of Erwinia chrysanthemi: the key role of SufC, an orphan ABC ATPase. Mol. Microbiol. 39, 960-972. (YOR171c) and LCB5 (YLR260w) genes of Saccharomyces encode sphingoid long chain base kinases. J. Biol. Chem. 273, 19437-19442.

Nagy, E., Losick, R., and Kahne, D. (2019). Robust suppression of lipopolysaccharide deficiency in Acinetobacter baumannii by growth in minimal medium. J. Bacteriol. 201, 420.

Nikaido, H. (2003). Molecular basis of bacterial outer membrane permeability revisited. Microbiol. Mol. Biol. Rev. 67, 593-656.

Olea-Ozuna, R.J., Poggio, S., Bergström, E., Quiroz-Rocha, E., García-Soriano, D.A., Sahonero-Canavesi, D.X., Padilla-Gómez, J., Martínez-Aguilar, L., López-Lara, I.M., ThomasOates, J., et al. (2021). Five structural genes required for ceramide synthesis in Caulobacter and for bacterial survival. Environ. Microbiol. 23, 143-159.

Peng, D., Hong, W., Choudhury, B.P., Carlson, R.W., and Gu, X.-X. (2005). Moraxella 7577. containing D-galacturonic acid that replaces phosphate residues. The structure of the lipid a of the lipopolysaccharide from the hyperthermophilic bacterium Aquifex pyrophilus. J. Biol. Chem. 275, 11222-11228. 
Qureshi, N., Takayama, K., Mascagni, P., Honovich, J., Wong, R., and Cotter, R.J. (1988). Complete structural determination of lipopolysaccharide obtained from deep rough mutant of Escherichia coli. Purification by high performance liquid chromatography and direct analysis by plasma desorption mass spectrometry. J. Biol. Chem. 263, 11971-11976.

Radolf, J.D., and Kumar, S. (2018). The Treponema pallidum outer membrane. Curr. Top. Microbiol. Immuno. 415, 1-38. D.B., Theriot, J.A., and Huang, K.C. (2018). The outer membrane is an essential load-bearing element in Gram-negative bacteria. Nature 559, 617-621.

Samuel, G., and Reeves, P. (2003). Biosynthesis of O-antigens: Genes and pathways involved in nucleotide sugar precursor synthesis and O-antigen assembly. Carboyhdr. Res. 338, 25032519. phosphatase homolog regulates cell separation, outer membrane integrity, and morphology in Caulobacter crescentus. J. Bacteriol. 193, 4361-4370.

da Silva Neto, J.F., Braz, V.S., Italiani, V.C.S., and Marques, M.V. (2009). Fur controls iron homeostasis and oxidative stress defense in the oligotrophic alpha-proteobacterium Caulobacter crescentus. Nucleic Acids Res. 37, 4812-4825. of Caulobacter crescentus to iron availability. BMC Genomics 14, 549. Due to Structural Support from Elongasome Peptidoglycan Synthesis. mBio 12, e0309921. component signal transduction pathways regulating growth and cell cycle progresion in a 1351 bacterium: A systems-level analysis. PLoS Biol. 3, e334. 
1353 Smit, J., Kaltashov, I.A., Cotter, R.J., Vinogradov, E., Perry, M.B., Haider, H., and Qureshi, N.

1354 (2008). Structure of a novel lipid A obtained from the lipopolysaccharide of Caulobacter

1355 crescentus. Innate Immun. 14, 25-37.

Stankeviciute, G., Guan, Z., Goldfine, H., and Klein, E.A. (2019). Caulobacter crescentus adapts to phosphate starvation by synthesizing anionic glycoglycerolipids and a novel glycosphingolipid. mBio 10, 107.

Stankeviciute, G., Tang, P., Ashley, B., Chamberlain, J.D., Hansen, M.E.B., Coleman, A., D'Emilia, R., Fu, L., Mohan, E.C., Nguyen, H., et al. (2021). Convergent evolution of bacterial ceramide synthesis. Nat. Chem. Biol. DOI: 10.1038/s41589-021-00948-7.

Steeghs, L., Hartog, R. den, Boer, A. den, Zomer, B., Roholl, P., and Ley, P. van der (1998). Meningitis bacterium is viable without endotoxin. Nature 392, 449-450.

Thanbichler, M., Iniesta, A.A., and Shapiro, L. (2007). A comprehensive set of plasmids for vanillate- and xylose-inducible gene expression in Caulobacter crescentus. Nucleic Acids Res. 35, e137.

Toh, E., Jr, H.D.K., and Brun, Y.V. (2008). Characterization of the Caulobacter crescentus holdfast polysaccharide biosynthesis pathway reveals significant redundancy in the initiating glycosyltransferase and polyerase steps. J. Bacteriol. 190, 7219-7131.

Velkov, T., Thompson, P.E., Nation, R.L., and Li, J. (2010). Structure--activity relationships of polymyxin antibiotics. J. Med. Chem. 53, 1898-1916. mutants of Caulobacter crescentus defective in surface attachment of the paracrystalline surface layer. J. Bacteriol. 176, 6313-6323.

Wang, X., Ribeiro, A.A., Guan, Z., McGrath, S.C., Cotter, R.J., and Raetz, C.R.H. (2006). Structure and biosynthesis of free lipid A molecules that replace lipopolysaccharide in Francisella tularensis subsp. novicida. Biochemistry 45, 14427-14440. and further applications of the procedure. Methods Carbohydr. Chem. 5, 83-91. 
Wetmore, K.M., Price, M.N., Waters, R.J., Lamson, J.S., He, J., Hoover, C.A., Blow, M.J., Bristow, J., Butland, G., Arkin, A.P., et al. (2015). Rapid quantification of mutant fitness in diverse bacteria by sequencing randomly bar-coded transposons. mBio 6, e00306-e00315.

Whitfield, C., and Trent, M.S. (2014). Biosynthesis and export of bacterial lipopolysaccharides. Annu. Rev. Biochem. 83, 99-128.

Wofford, J.D., Bolaji, N., Dziuba, N., Outten, F.W., and Lindahl, P.A. (2019). Evidence that a respiratory shield in Escherichia coli protects a low-molecular-mass Fell pool from O2dependent oxidation. J. Biol. Chem. 294, 50-62.

Yeowell, H.N., and White, J.R. (1982). Iron requirement in the bactericidal mechanism of streptonigrin. Antimicrob. Agents Chemother. 22, 961-968.

1403

Yoon, S.H., Liang, T., Schneider, T., Oyler, B.L., Chandler, C.E., Ernst, R.K., Yen, G.S., Huang,

1405 Y., Nilsson, E., and Goodlett, D.R. (2016). Rapid lipid a structure determination via surface 1406 acoustic wave nebulization and hierarchical tandem mass spectrometry algorithm. Rapid 1407 Commun. Mass Spectrom. 30, 2555-2560. Gram-negative bacteria. Curr. Opin. Microbiol. 16, 779-785.

1413 MotionCor2: anisotropic correction of bean-induced motion for improved cryo-electron

1414 microscopy. Nat. Methods 14, 331-332. during the Caulobacter cell cycle. PLoS Genet. 11, e1004831. 


\section{Supplementary Tables}

1422

\section{Table S1, related to Figure 1: Single-nucleotide polymorphisms and indels in $\Delta c t p A$}

\section{4 suppressors ${ }^{\mathrm{a}}$}

1425

\begin{tabular}{|c|c|c|c|c|c|}
\hline Strain & $\begin{array}{l}\text { Sequence } \\
\text { position }^{b}\end{array}$ & Gene $^{c}$ & Annotation & $\begin{array}{l}\text { Base } \\
\text { Change }\end{array}$ & $\begin{array}{l}\text { Amino Acid } \\
\text { Substitution }\end{array}$ \\
\hline \multirow[t]{2}{*}{ S3 } & 1668695 & 01553 & $\begin{array}{l}\text { Undecaprenyl-phosphate beta-N-acetyl-D- } \\
\text { fucosaminephosphotrans- } \\
\text { ferase }\end{array}$ & $\mathrm{G}>\mathrm{T}$ & $\mathrm{K} 54 \mathrm{~N}$ \\
\hline & 2383967 & 02235 & SGNH hydrolase family protein & $\mathrm{G}>\mathrm{A}$ & G109D \\
\hline S8 & 3492550 & 03316 & $\begin{array}{l}\text { UDP-N-acetylglucosamine 4,6- } \\
\text { dehydratase/UDP-D-quinovosamine 4- } \\
\text { dehydrogenase }\end{array}$ & AG $>$ ATG & E487Frameshift \\
\hline S16 & 58496 & 00055 & Ferric uptake regulation protein & $A>T$ & L38Q \\
\hline \multirow[t]{2}{*}{ S21 } & 515085 & 00497 & Putative rhamnosyl transferase & $\mathrm{G}>\mathrm{A}$ & G263D \\
\hline & 1378399 & 01250 & FecCD-family transporter protein & $\mathrm{GCC}>\mathrm{GC}$ & A340Frameshift \\
\hline \multirow[t]{3}{*}{ S32 } & 1157376 & 01056 & Methyltransferase & $A>A G$ & S31Framshift \\
\hline & 2164248 & 02016 & nuoMNADH-quinone oxidoreductase chain M & $\mathrm{C}>\mathrm{T}$ & M7I \\
\hline & 2949436 & 02792 & TonB-dependent outer membrane receptor & $A>G$ & S516G \\
\hline S36 & 3901011 & 03733 & Mannose-1-phosphate guanylyltransferase & $\mathrm{C}>\mathrm{T}$ & W279Stop \\
\hline S38 & 726779 & 00669 & Glycosyltransferase family 99 protein WbsX & $\mathrm{CTG}>\mathrm{CG}$ & Q476Frameshift \\
\hline \multirow[t]{3}{*}{ S40 } & 378822 & 00362 & Zinc uptake regulation protein & $A>T$ & C156S \\
\hline & 2868272 & - & - & $\mathrm{G}>\mathrm{C}$ & - \\
\hline & 3913883 & 03744 & dTDP-glucose 4,6-dehydratase & $\mathrm{T}>\mathrm{A}$ & I269F \\
\hline \multirow[t]{3}{*}{ S43 } & 1377138 & 01249 & ABC-transporter substrate binding protein & $\mathrm{C}>\mathrm{T}$ & A169V \\
\hline & 1668861 & 01553 & $\begin{array}{l}\text { Undecaprenyl-phosphate beta-N-acetyl-D- } \\
\text { fucosaminephosphotransferase }\end{array}$ & $\mathrm{CC}>\mathrm{GA}$ & P110E \\
\hline & 2976030 & 02820 & TadG-family protein & $\mathrm{C}>\mathrm{T}$ & Silent \\
\hline S44 & 727833 & 00669 & Glycosyltransferase family 99 protein WbsX & $A>C$ & L125R \\
\hline \multirow[t]{2}{*}{ S47 } & 537654 & 00524 & Conserved hypothetical cytosolic protein & $A>A G$ & L369Frameshift \\
\hline & 1173251 & 01068 & Glycosyltransferase & $\mathrm{GCC}>\mathrm{GC}$ & R293Frameshift \\
\hline \multirow[t]{3}{*}{ S52 } & 514656 & 00497 & Putative rhamnosyl transferase & $T>G$ & L120R \\
\hline & 810384 & 00752 & 3-hydroxybutyryl-CoA dehydrogenase & $\mathrm{T}>\mathrm{A}$ & Stop>Y \\
\hline & 3797776 & - & - & $\mathrm{T}>\mathrm{C}$ & - \\
\hline S53 & 58379 & 00055 & Ferric uptake regulation protein & $\mathrm{G}>\mathrm{T}$ & S77Stop \\
\hline
\end{tabular}




\begin{tabular}{|c|c|c|c|c|c|}
\hline & 1778110 & 01656 & $\begin{array}{l}\text { Endonuclease/exonuclease/phosphatase } \\
\text { family protein }\end{array}$ & $A>C$ & L4R \\
\hline & 3900664 & 03733 & Mannose-1-phosphate guanylyltransferase & $\mathrm{G}>\mathrm{A}$ & Q395Stop \\
\hline \multirow[t]{2}{*}{ S54 } & 1377127 & 01249 & ABC-transporter substrate binding protein & $\mathrm{CGG}>\mathrm{CG}$ & G166Frameshift \\
\hline & 3492771 & 03316 & $\begin{array}{l}\text { UDP-N-acetylglucosamine 4,6- } \\
\text { dehydratase/UDP-D-quinovosamine 4- } \\
\text { dehydrogenase }\end{array}$ & $\mathrm{G}>\mathrm{A}$ & E561K \\
\hline \multirow[t]{2}{*}{ S57 } & 1492703 & 01378 & Protein-L-isoaspartate O-methyltransferase & $\mathrm{G}>\mathrm{GC}$ & G59Frameshift \\
\hline & 2487074 & 02347 & Phosphomannomutase/phosphoglucomutase & $\mathrm{G}>\mathrm{A}$ & G266D \\
\hline \multirow[t]{2}{*}{ S111 } & 295119 & 00283 & $\begin{array}{l}\text { 2,3,4,5-tetrahydropyridine-2,6-dicarboxylate } \\
\text { N-succinyltransferase }\end{array}$ & $A>T$ & L284Q \\
\hline & 1173965 & 01068 & Glycosyltransferase & $\mathrm{TGC}>\mathrm{T}$ & R55Frameshift \\
\hline \multirow[t]{4}{*}{ S112 } & 311705 & 00297 & Two-component response regulator & $\mathrm{C}>\mathrm{T}$ & W64Stop \\
\hline & 315570 & 00301 & Phosphotransferase family protein & $T>G$ & I319S \\
\hline & 2487307 & 02347 & Phosphomannomutase/phosphoglucomutase & $\mathrm{G}>\mathrm{A}$ & E344K \\
\hline & 3569520 & 03399 & Flavin prenyltransferase UbiX & $\mathrm{C}>\mathrm{T}$ & A159T \\
\hline
\end{tabular}

\section{6}

$1427{ }^{\text {a } I n}$ addition to the indicated mutations, each strain is also $\triangle \operatorname{ctp} A \Delta s s p$.

1428 bequence positions refer to the genome of Caulobacter crescentus NA1000 (NC_011916.1).

1429 'The prefix for each gene number is CCNA_.

\section{0}


1431 Table S2, related to Figure 3: Single-nucleotide polymorphisms and indels identified in $1432 \Delta / p x C$ suppressors ${ }^{a}$

1433

1434

\begin{tabular}{|l|l|l|l|l|l|}
\hline Strain & $\begin{array}{l}\text { Sequence } \\
\text { position }^{\mathrm{b}}\end{array}$ & Gene $^{\mathrm{c}}$ & Annotation & $\begin{array}{l}\text { Base } \\
\text { Change }\end{array}$ & $\begin{array}{l}\text { Amino Acid } \\
\text { Substitution }\end{array}$ \\
\hline S1 & 58446 & 00055 & Ferric uptake regulation protein Fur & A>T & Y55N \\
\hline & 4000704 & 03835 & 3-oxoacyl-(Acyl-carrier-protein) synthase & C>T & G396S \\
\hline S5 & 58436 & 00055 & Ferric uptake regulation protein Fur & A>T & V58E \\
\hline
\end{tabular}

1435

1436 aln addition to the indicated mutations, each strain is also $\Delta / p x C \Delta s s p B \Delta 00497$.

1437 bequence positions refer to the genome of Caulobacter crescentus NA1000 (NC_011916.1).

$1438{ }^{\mathrm{c}}$ The prefix for each gene number is CCNA_. 
1440 Table S3, related to Figure 3: Growth rates in PYE medium at $22^{\circ} \mathrm{C}$.

1441

1442

\begin{tabular}{|l|c|c|}
\hline & \multicolumn{2}{|c|}{$\begin{array}{r}\text { Doubling time +/- standard } \\
\text { deviation (hours) }\end{array}$} \\
\hline Strain & PYE $30^{\circ} \mathrm{C}$ & PYE 22 ${ }^{\circ} \mathrm{C}$ \\
\hline NA1000 & $1.61+/-0.04$ & $2.50+/-0.17$ \\
\hline$\Delta s s p B$ & $1.51+/-0.04$ & $2.44+/-0.09$ \\
\hline$\Delta$ fur $\Delta s s p B$ & $1.77+/-0.08$ & $3.16+/-0.21$ \\
\hline
\end{tabular}

1443 aDoubling times were calculated from optical density measurements $(660 \mathrm{~nm})$ during 1444 exponential growth.

1445

1446 
Table S4, related to STAR Methods: Strains used in this study.

\begin{tabular}{|c|c|c|c|c|}
\hline $\begin{array}{l}\text { Strain } \\
\text { Number }\end{array}$ & Description & Note & $\begin{array}{l}\text { NCBI BioSample } \\
\text { Accessions }\end{array}$ & Reference \\
\hline KR4000 & Wild-type Caulobacter NA1000 & & & $\begin{array}{r}\text { (Evinger and } \\
\text { Agabian, } \\
1977)\end{array}$ \\
\hline KR3180 & NA1000 pJS14 & & & This study \\
\hline KR1499 & $\Delta s s p B:: a a d A$ & & & $\begin{array}{r}\text { (Shapland et } \\
\text { al., 2011) }\end{array}$ \\
\hline KR3877 & $\triangle C C N A \_00497::$ hyg & & & This study \\
\hline KR4198 & $\triangle C C N A \_00497:: h y g$ pJS14 & & & This study \\
\hline KR3871 & $\triangle C C N A \_01553::$ hyg & & & This study \\
\hline KR4197 & $\triangle C C N A$ 01553::hyg pJS14 & & & This study \\
\hline KR4076 & $\Delta$ fur::hyg & & & This study \\
\hline KR4199 & $\Delta$ fur::hyg pJS14 & & & This study \\
\hline KR3953 & $\triangle C C N A$ A0497::hyg $\triangle s s p B:: a a d A$ & & & This study \\
\hline KR4115 & $\triangle C C N A$ A1055::hyg $\triangle s s p B:: a a d A$ & & & This study \\
\hline KR4116 & $\triangle C C N A \_01068::$ hyg $\triangle s s p B:: a a d A$ & & & This study \\
\hline KR3954 & $\triangle C C N A \_01553::$ hyg $\triangle s s p B:: a a d A$ & & & This study \\
\hline KR3955 & $\triangle C C N A$ A3733::hyg $\triangle s s p B:: a a d A$ & & & This study \\
\hline KR4077 & $\Delta$ fur::hyg $\Delta s s p B:: a a d A$ & & & This study \\
\hline KR4153 & $\Delta s s p B:: a a d A$ pXCERN-2 & & & This study \\
\hline KR4154 & $\begin{array}{l}\triangle C C N A \_00497:: \text { hyg } \triangle s s p B:: a a d A \\
\text { pXCERN-2 }\end{array}$ & & & This study \\
\hline KR4155 & $\begin{array}{l}\triangle C C N A \_01553:: \text { hyg } \triangle s s p B:: a a d A \\
\text { pXCERN-2 }\end{array}$ & & & This study \\
\hline KR4156 & $\begin{array}{l}\triangle C C N A \_03733:: \text { hyg } \triangle s s p B:: a a d A \\
\text { pXCERN-2 }\end{array}$ & & & This study \\
\hline KR4157 & $\Delta$ fur::hyg $\Delta s s p B:: a a d A$ pXCERN-2 & & & This study \\
\hline KR4158 & $\begin{array}{l}\triangle C C N A \_00497:: \text { hyg } \triangle s s p B:: a a d A \\
\text { pZIK172 }\end{array}$ & & & This study \\
\hline KR4159 & $\begin{array}{l}\triangle C C N A \text { 01553::hyg } \triangle s s p B:: a a d A \\
\text { pZIK173 }\end{array}$ & & & This study \\
\hline
\end{tabular}




\begin{tabular}{|c|c|c|c|c|}
\hline KR4160 & $\begin{array}{l}\triangle C C N A \_03733:: \text { hyg } \triangle s s p B:: a a d A \\
\text { pZIK174 }\end{array}$ & & & This study \\
\hline KR4161 & $\Delta$ fur::hyg $\Delta s s p B:: a a d A$ pZIK175 & & & This study \\
\hline KR4269 & $\Delta$ fur::hyg $\Delta s s p B:: a a d A$ pZIK197 & & & This study \\
\hline KR3906 & $\Delta c t p A:: t e t A R \Delta s s p B:: a a d A$ pAB6 & $\begin{array}{l}\text { Restock of KR2423 } \\
\text { from Shapland } 2011\end{array}$ & & $\begin{array}{r}\text { (Shapland et } \\
\text { al., 2011) }\end{array}$ \\
\hline KR4111 & $\begin{array}{l}\Delta c t p A:: t e t A R \Delta s s p B:: a a d A \\
\Delta C C N A \_00497:: h y g \text { pAB6 }\end{array}$ & & SAMN12568762 & This study \\
\hline KR4112 & $\begin{array}{l}\Delta c t p A:: t e t A R \Delta s s p B:: a a d A \\
\Delta C C N A \_01553:: h y g \text { pAB6 }\end{array}$ & & SAMN12568763 & This study \\
\hline KR4092 & $\begin{array}{l}\operatorname{ctpA}:: \text { tetAR } \triangle s s p B:: \text { aadA } \\
\triangle C C N A \_03733:: \text { hyg pAB6 }\end{array}$ & & SAMN12568761 & This study \\
\hline KR4090 & $\begin{array}{l}\triangle \operatorname{ctp} A:: \text { tetAR } \Delta s s p B:: a a d A \\
\Delta \text { fur::hyg pAB6 }\end{array}$ & & SAMN12568759 & This study \\
\hline KR4007 & $\Delta / p x C:: t e t A R \Delta s s p B:: a a d A$ pZIK133 & & & This study \\
\hline KR4008 & $\begin{array}{l}\Delta / p \times C:: \text { tetAR } \triangle s s p B:: a a d A \\
\triangle C C N A \_00497:: \text { hyg pZIK133 }\end{array}$ & & & This study \\
\hline KR4223 & $\begin{array}{l}\Delta / p \times C:: t e t A R \triangle C C N A \_00497:: h y g \\
\text { pZIK133 }\end{array}$ & $\begin{array}{l}\text { Parent strain for } \\
\text { isolating KR4224 and } \\
\text { KR4225 }\end{array}$ & & This study \\
\hline KR4009 & $\begin{array}{l}\Delta / p \times C:: \text { tetAR } \triangle s s p B:: \text { aadA } \\
\Delta C C N A \_01553:: \text { hyg pZIK133 }\end{array}$ & & & This study \\
\hline KR4010 & $\begin{array}{l}\Delta / p \times C:: t e t A R \Delta s s p B:: a a d A \\
\Delta C C N A \_03733:: \text { hyg pZIK133 }\end{array}$ & & & This study \\
\hline KR4091 & $\begin{array}{l}\Delta / p \times C:: t e t A R \Delta s s p B:: a a d A \\
\Delta f u r:: h y g \text { pZIK133 }\end{array}$ & & SAMN12568760 & This study \\
\hline KR4113 & 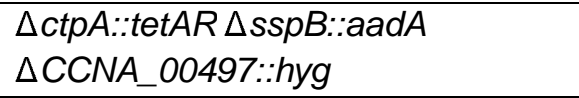 & & SAMN12568767 & This study \\
\hline KR4114 & $\begin{array}{l}\Delta c t p A:: t e t A R \Delta s s p B:: a a d A \\
\triangle C C N A \_01553:: h y g\end{array}$ & & SAMN12568768 & This study \\
\hline KR4104 & $\begin{array}{l}\triangle c t p A:: t e t A R \Delta s s p B:: a a d A \\
\triangle C C N A \_03733:: \text { hyg }\end{array}$ & & SAMN12568766 & This study \\
\hline KR4102 & $\begin{array}{l}\Delta c t p A:: t e t A R \Delta s s p B:: a a d A \\
\Delta \text { fur::hyg }\end{array}$ & & SAMN12568764 & This study \\
\hline KR4103 & $\begin{array}{l}\Delta l p x C:: t e t A R \Delta s s p B:: a a d A \\
\Delta \text { fur::hyg }\end{array}$ & & SAMN12568765 & This study \\
\hline KR4176 & $\begin{array}{l}\Delta \operatorname{ctp} A:: t e t A R \Delta s s p B:: a a d A \\
\Delta \text { fur::hyg pZIK175 }\end{array}$ & & & This study \\
\hline KR4270 & $\begin{array}{l}\Delta \operatorname{ctp} A:: t e t A R \Delta s s p B:: a a d A \\
\Delta \text { fur::hyg pZIK197 }\end{array}$ & & & This study \\
\hline KR4177 & 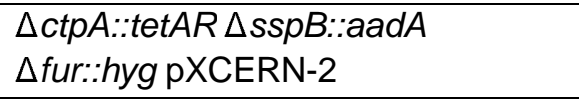 & & & This study \\
\hline KR4178 & $\begin{array}{l}\Delta / p x C:: \text { tetAR } \Delta s s p B:: a a d A \\
\Delta \text { fur::hyg pZIK175 }\end{array}$ & & & This study \\
\hline
\end{tabular}




\begin{tabular}{|c|c|c|c|}
\hline KR4271 & $\begin{array}{l}\Delta / p \times C:: t e t A R \Delta s s p B:: a a d A \\
\Delta f u r:: h y g \text { pZIK197 }\end{array}$ & & This study \\
\hline KR4179 & $\begin{array}{l}\Delta / p \times C:: t e t A R \Delta s s p B:: a a d A \\
\Delta f u r:: h y g \text { pXCERN-2 }\end{array}$ & & This study \\
\hline KR4180 & $\begin{array}{l}\triangle c t p A:: \text { tetAR } \triangle s s p B:: \text { aadA } \\
\triangle C C N A \_03733:: \text { hyg pZIK174 }\end{array}$ & & This study \\
\hline KR4181 & $\begin{array}{l}\triangle c t p A:: t e t A R \Delta s s p B:: \text { aad } A \\
\triangle C C N A \_03733:: \text { hyg pXCERN-2 }\end{array}$ & & This study \\
\hline KR4182 & $\begin{array}{l}\Delta c t p A:: t e t A R \Delta s s p B:: a a d A \\
\Delta C C N A \_00497:: \text { hyg pZIK172 }\end{array}$ & & This study \\
\hline KR4183 & $\begin{array}{l}\Delta c t p A:: t e t A R \Delta s s p B:: \text { aadA } \\
\Delta C C N A \_00497:: \text { hyg pXCERN-2 }\end{array}$ & & This study \\
\hline KR4148 & $\begin{array}{l}\Delta / p x C:: t e t A R \Delta s s p B:: a a d A \\
\Delta \text { fur::hyg pZIK133 }\end{array}$ & $\begin{array}{l}\text { Re-introduction of } \\
\text { plasmid for } \\
\text { complementation }\end{array}$ & This study \\
\hline KR4149 & $\begin{array}{l}\Delta / p x C:: \text { tetAR } \Delta s s p B:: a a d A \\
\Delta \text { fur::hyg pJS14 }\end{array}$ & & This study \\
\hline KR4150 & $\begin{array}{l}\Delta c t p A:: t e t A R \Delta s s p B:: a a d A \\
\triangle C C N A \_03733:: \text { hyg pAB6 }\end{array}$ & $\begin{array}{l}\text { Re-introduction of } \\
\text { plasmid for } \\
\text { complementation }\end{array}$ & This study \\
\hline KR4151 & $\begin{array}{l}\triangle c t p A:: t e t A R \Delta s s p B:: a a d A \\
\triangle C C N A \_03733:: \text { hyg pJS14 }\end{array}$ & & This study \\
\hline KR4264 & NA1000 pZIK200 & & This study \\
\hline KR4147 & NA1000 pZIK171 & & This study \\
\hline KR4170 & NA1000 pZIK179 & & This study \\
\hline KR4489 & $\triangle C C N A \_01217$ & & This study \\
\hline KR4549 & $\triangle C C N A \_01217$ vanA::01217::FLAG & pKR438 integrated & This study \\
\hline KR4450 & $\triangle C C N A \_01218$ & & This study \\
\hline KR4505 & $\triangle C C N A \_01218 \operatorname{vanA}:: 01218:: F L A G$ & pKR435 integrated & This study \\
\hline KR4430 & $\triangle C C N A \_01219$ & & This study \\
\hline KR4501 & $\triangle C C N A \_01219$ vanA::01219::FLAG & pKR436 integrated & This study \\
\hline $\begin{array}{l}\text { EK720 } \\
\text { (KR443 } \\
1)\end{array}$ & $\triangle C C N A \_01220$ & & $\begin{array}{r}\text { (Stankeviciute } \\
\text { et al., 2019) }\end{array}$ \\
\hline KR4530 & $\triangle C C N A \_01220$ vanA::01220::FLAG & pKR437 integrated & This study \\
\hline KR4517 & $\begin{array}{l}\Delta \text { fur::hyg } \triangle s s p B:: a a d A \\
\triangle C C N A \_01217\end{array}$ & & This study \\
\hline
\end{tabular}




\begin{tabular}{|c|c|c|c|c|}
\hline KR4551 & $\begin{array}{l}\Delta \text { fur::hyg } \triangle s s p B:: \text { aadA } \\
\triangle \text { CCNA_01217 vanA::01217::FLAG }\end{array}$ & pKR438 integrated & & This study \\
\hline KR4442 & $\begin{array}{l}\Delta \text { fur::hyg } \Delta s s p B:: a a d A \\
\triangle C C N A \_01218\end{array}$ & & & This study \\
\hline KR4503 & $\begin{array}{l}\Delta \text { fur::hyg } \Delta s s p B:: a a d A \\
\triangle C C N A \_01218 \text { vanA::01218::FLAG }\end{array}$ & pKR435 integrated & & This study \\
\hline KR4438 & $\begin{array}{l}\Delta \text { fur::hyg } \Delta s s p B:: a a d A \\
\triangle C C N A \_01219\end{array}$ & & & This study \\
\hline KR4513 & $\begin{array}{l}\Delta \text { fur::hyg } \Delta s s p B:: a a d A \\
\triangle C C N A \text { 01219 vanA::01219::FLAG }\end{array}$ & pKR436 integrated & & This study \\
\hline KR4439 & $\begin{array}{l}\Delta \text { fur::hyg } \Delta s s p B:: a a d A \\
\triangle C C N A \_01220\end{array}$ & & & This study \\
\hline KR4532 & $\begin{array}{l}\Delta \text { fur::hyg } \Delta s s p B:: a a d A \\
\triangle C C N A \_01220 \text { vanA::01220::FLAG }\end{array}$ & pKR437 integrated & & This study \\
\hline KR4509 & $\begin{array}{l}\Delta l p x C:: \text { tetAR } \Delta s s p B:: a a d A \\
\Delta \text { fur::hyg } \triangle C C N A \_01217 \text { pZIK133 }\end{array}$ & & & This study \\
\hline KR4555 & $\begin{array}{l}\Delta / p x C:: t e t A R \triangle s s p B:: a a d A \\
\Delta \text { fur::hyg } \triangle C C N A=01217 \\
\text { vanA::01217::FLAG pZIK133 }\end{array}$ & pKR438 integrated & & This study \\
\hline KR4451 & $\begin{array}{l}\Delta / p \times C:: \text { tetAR } \triangle s s p B:: a a d A \\
\Delta \text { fur::hyg } \triangle C C N A \_01218 \text { pZIK133 }\end{array}$ & & & This study \\
\hline KR4527 & $\begin{array}{l}\Delta / p \times C:: t e t A R \triangle s s p B:: a a d A \\
\Delta \text { fur::hyg } \triangle C C N A \text { 01218 } \\
\text { vanA::01218::FLAG pZIK133 }\end{array}$ & pKR435 integrated & & This study \\
\hline KR4441 & $\begin{array}{l}\Delta l p x C:: \text { tetAR } \triangle s s p B:: \text { aadA } \\
\Delta \text { fur::hyg } \triangle C C N A \_01220 \text { pZIK133 }\end{array}$ & & & This study \\
\hline KR4545 & $\begin{array}{l}\Delta / p \times C:: \text { tetAR } \triangle s s p B:: a a d A \\
\Delta \text { fur::hyg } \triangle C C N A=01220 \\
\text { vanA::01220::FLAG pZIK133 }\end{array}$ & pKR437 integrated & & This study \\
\hline KR4205 & $\begin{array}{l}\Delta c t p A:: t e t A R \triangle s s p B:: a a d A \\
\text { suppressor isolate \#3 }\end{array}$ & $\begin{array}{l}\text { Additional genotype in } \\
\text { Supplementary Table } 1\end{array}$ & SAMN11107060 & This study \\
\hline KR4206 & $\begin{array}{l}\triangle c t p A:: t e t A R \Delta s s p B:: a a d A \\
\text { suppressor isolate \#8 }\end{array}$ & $\begin{array}{l}\text { Additional genotype in } \\
\text { Supplementary Table } 1\end{array}$ & SAMN11107061 & This study \\
\hline KR4207 & $\begin{array}{l}\Delta c t p A:: t e t A R \Delta s s p B:: a a d A \\
\text { suppressor isolate \#16 }\end{array}$ & $\begin{array}{l}\text { Additional genotype in } \\
\text { Supplementary Table } 1\end{array}$ & SAMN11107062 & This study \\
\hline KR4208 & $\begin{array}{l}\triangle \operatorname{ctp} A:: t e t A R \Delta s s p B:: a a d A \\
\text { suppressor isolate \#21 }\end{array}$ & $\begin{array}{l}\text { Additional genotype in } \\
\text { Supplementary Table } 1\end{array}$ & SAMN11107063 & This study \\
\hline KR4209 & $\begin{array}{l}\Delta c t p A:: t e t A R \Delta s s p B:: a a d A \\
\text { suppressor isolate \#32 }\end{array}$ & $\begin{array}{l}\text { Additional genotype in } \\
\text { Supplementary Table } 1\end{array}$ & SAMN11107064 & This study \\
\hline KR4210 & $\begin{array}{l}\Delta c t p A:: t e t A R \Delta s s p B:: a a d A \\
\text { suppressor isolate \#36 }\end{array}$ & $\begin{array}{l}\text { Additional genotype in } \\
\text { Supplementary Table } 1\end{array}$ & SAMN11107065 & This study \\
\hline KR4211 & $\begin{array}{l}\Delta c t p A:: t e t A R \Delta s s p B:: a a d A \\
\text { suppressor isolate \#38 }\end{array}$ & $\begin{array}{l}\text { Additional genotype in } \\
\text { Supplementary Table } 1\end{array}$ & SAMN11107066 & This study \\
\hline KR4212 & $\begin{array}{l}\Delta c t p A:: t e t A R \Delta s s p B:: a a d A \\
\text { suppressor isolate \#40 }\end{array}$ & $\begin{array}{l}\text { Additional genotype in } \\
\text { Supplementary Table } 1\end{array}$ & SAMN11107067 & This study \\
\hline KR4213 & $\begin{array}{l}\triangle \operatorname{ctp} A:: t e t A R \Delta s s p B:: a a d A \\
\text { suppressor isolate } \# 43\end{array}$ & $\begin{array}{l}\text { Additional genotype in } \\
\text { Supplementary Table } 1\end{array}$ & SAMN11107068 & This study \\
\hline
\end{tabular}




\begin{tabular}{|c|c|c|c|c|}
\hline KR4214 & $\begin{array}{l}\triangle c t p A:: t e t A R \triangle s s p B:: a a d A \\
\text { suppressor isolate \#44 }\end{array}$ & $\begin{array}{l}\text { Additional genotype in } \\
\text { Supplementary Table } 1\end{array}$ & SAMN11107069 & This study \\
\hline KR4215 & $\begin{array}{l}\triangle \operatorname{ctp} A:: t e t A R \Delta s s p B:: a a d A \\
\text { suppressor isolate \#47 }\end{array}$ & $\begin{array}{l}\text { Additional genotype in } \\
\text { Supplementary Table } 1\end{array}$ & SAMN11107070 & This study \\
\hline KR4216 & $\begin{array}{l}\Delta c t p A:: t e t A R \Delta s s p B:: a a d A \\
\text { suppressor isolate } \# 52\end{array}$ & $\begin{array}{l}\text { Additional genotype in } \\
\text { Supplementary Table } 1\end{array}$ & SAMN11107071 & This study \\
\hline KR4217 & $\begin{array}{l}\Delta c t p A:: t e t A R \Delta s s p B:: a a d A \\
\text { suppressor isolate } \# 53\end{array}$ & $\begin{array}{l}\text { Additional genotype in } \\
\text { Supplementary Table } 1\end{array}$ & SAMN11107072 & This study \\
\hline KR4218 & $\begin{array}{l}\Delta c t p A:: t e t A R \Delta s s p B:: a a d A \\
\text { suppressor isolate } \# 54\end{array}$ & $\begin{array}{l}\text { Additional genotype in } \\
\text { Supplementary Table } 1\end{array}$ & SAMN11107073 & This study \\
\hline KR4219 & $\begin{array}{l}\Delta c t p A:: t e t A R \Delta s s p B:: a a d A \\
\text { suppressor isolate } \# 57\end{array}$ & $\begin{array}{l}\text { Additional genotype in } \\
\text { Supplementary Table } 1\end{array}$ & SAMN11107074 & This study \\
\hline KR4220 & $\begin{array}{l}\Delta c t p A:: t e t A R \Delta s s p B:: a a d A \\
\text { suppressor isolate } \# 111\end{array}$ & $\begin{array}{l}\text { Additional genotype in } \\
\text { Supplementary Table } 1\end{array}$ & SAMN11107075 & This study \\
\hline KR4221 & $\begin{array}{l}\Delta c t p A:: t e t A R \Delta s s p B:: a a d A \\
\text { suppressor isolate } \# 112\end{array}$ & $\begin{array}{l}\text { Additional genotype in } \\
\text { Supplementary Table } 1\end{array}$ & SAMN11107076 & This study \\
\hline KR4224 & $\begin{array}{l}\Delta / p x C:: t e t A R \Delta s s p B:: a a d A \\
\text { suppressor isolate \#1 }\end{array}$ & $\begin{array}{l}\text { Additional genotype in } \\
\text { Supplementary Table } 2\end{array}$ & SAMN11107077 & This study \\
\hline KR4225 & $\begin{array}{l}\Delta / p \times C:: t e t A R \Delta s s p B:: a a d A \\
\text { suppressor isolate \#5 }\end{array}$ & $\begin{array}{l}\text { Additional genotype in } \\
\text { Supplementary Table } 2\end{array}$ & SAMN11107078 & This study \\
\hline
\end{tabular}


Table S5, related to STAR Methods: Plasmids used in this study.

\begin{tabular}{|c|c|c|}
\hline Name & Description & Reference \\
\hline pJS14 & $\begin{array}{l}\text { Broad host-range cloning vector; high copy; } \text { chlor }^{\mathrm{H}} \text {; pBBR1MCS derivative } \\
\text { with unique EcoRI site }\end{array}$ & $\begin{array}{r}\text { (J. Skerker, } \\
\text { unpublished) }\end{array}$ \\
\hline pNPTS138 & $\mathrm{kan}^{\mathrm{R}}$; sacB-containing integration vector & $\begin{array}{r}\text { (M.R. Alley, } \\
\text { unpublished) }\end{array}$ \\
\hline pXCERN-2 & $\begin{array}{l}\text { For integration at } \mathrm{P}_{x y l x} ; \text { encodes xylose-inducible cerulean that can be } \\
\text { exhcanged for gene of interest; } \operatorname{kan}^{\mathrm{R}}\end{array}$ & $\begin{array}{l}\text { (Thanbichler } \\
\text { et al., 2007) }\end{array}$ \\
\hline pMCS-2 & For integration at locus specified by insert sequence; kan $^{\mathrm{H}}$ & $\begin{array}{l}\text { (Thanbichler } \\
\text { et al., 2007) }\end{array}$ \\
\hline pAB6 & pJS14-P $\mathrm{P}_{x y \mid x-c t p A-3 x F L A G-s s r A}$ & $\begin{array}{r}\text { (Shapland et } \\
\text { al., 2011) }\end{array}$ \\
\hline pZIK133 & pJS14-P ${ }_{x y \mid X-I p x C-3 x F L A G-s s r A}$ & This study \\
\hline pZIK172 & pXCERN-2-P ${ }_{x y \mid X}-C C N A \_00497$ & This study \\
\hline pZIK173 & pXCERN-2-P ${ }_{x y I X}-C C N A \_01553$ & This study \\
\hline pZIK174 & pXCERN-2-P ${ }_{x y I X}-C C N A \_03733$ & This study \\
\hline pZIK175 & pXCERN-2-P ${ }_{x y l x}-C C N A \_00055$ (fur) & This study \\
\hline pZIK78 & $\begin{array}{l}\text { pNPTS138-CCNA_00497::hyg; for replacing CCNA_00497 with } \\
\text { hygromycin resistance cassette }\end{array}$ & This study \\
\hline pZIK82 & $\begin{array}{l}\text { pNPTS138-CCNA_01055::hyg; for replacing CCNA_01055 with } \\
\text { hygromycin resistance cassette }\end{array}$ & This study \\
\hline pZIK81 & $\begin{array}{l}\text { pNPTS138-CCNA_01068::hyg; for replacing CCNA_01068 with } \\
\text { hygromycin resistance cassette }\end{array}$ & This study \\
\hline pZIK73 & $\begin{array}{l}\text { pNPTS138-CCNA_01553::hyg; for replacing CCNA_01553 with } \\
\text { hygromycin resistance cassette }\end{array}$ & This study \\
\hline pZIK80 & $\begin{array}{l}\text { pNPTS138-CCNA_03733::hyg; for replacing CCNA_03733 with } \\
\text { hygromycin resistance cassette }\end{array}$ & This study \\
\hline pZIK161 & $\begin{array}{l}\text { pNPTS138-CCNA_00055::hyg; for replacing fur with hygromycin resistance } \\
\text { cassette }\end{array}$ & This study \\
\hline pZIK134 & $\begin{array}{l}\text { pNPTS138-CCNA_02064::tetAR; for replacing IpxC with tetracycline } \\
\text { resistance cassette }\end{array}$ & This study \\
\hline 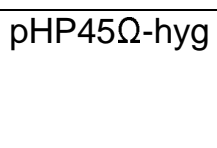 & For isolating hyg fragment; hyg $^{\mathrm{R}} ; \mathrm{amp}^{\mathrm{R}}$ & $\begin{array}{r}\text { (Blondelet- } \\
\text { Rouault et al., } \\
1997)\end{array}$ \\
\hline pKOC3 & Contains tetAR flanked by EcoRI sites; $\mathrm{amp}^{\mathrm{H}}$; tet $^{\mathrm{H}}$ & $\begin{array}{r}\text { (Skerker et al., } \\
\text { 2005) }\end{array}$ \\
\hline pVMCS-4 & For amplification of aacC1; gent ${ }^{\mathrm{H}}$ & $\begin{array}{l}\text { (Thanbichler } \\
\text { et al., 2007) }\end{array}$ \\
\hline pMCS-4 & For single integration to disrupt genomic loci; gent ${ }^{\mathrm{H}}$ & $\begin{array}{l}\text { (Thanbichler } \\
\text { et al., 2007) }\end{array}$ \\
\hline pVGFPC-2 & Complementation vector for expressing target genes from vanA locus; kan $^{\mathrm{H}}$ & $\begin{array}{l}\text { (Thanbichler } \\
\text { et al., 2007) }\end{array}$ \\
\hline
\end{tabular}




\begin{tabular}{|c|c|c|}
\hline pVGFPC-4 & $\begin{array}{l}\text { Complementation vector for expressing target genes from vanA locus; } \\
\text { gent }{ }^{R}\end{array}$ & $\begin{array}{l}\text { (Thanbichler } \\
\text { et al., 2007) }\end{array}$ \\
\hline pVCHYC-5 & Complementation vector for expressing target genes from vanA locus; tet ${ }^{\mathrm{B}}$ & $\begin{array}{l}\text { (Thanbichler } \\
\text { et al., 2007) }\end{array}$ \\
\hline pFLGC-1 & $\begin{array}{l}\text { Vector for adding FLAG tag to the C-terminus of open reading frames; } \\
\text { spec }^{R}\end{array}$ & $\begin{array}{l}\text { (Thanbichler } \\
\text { et al., 2007) }\end{array}$ \\
\hline pGS74 & pNPTS138-based plasmid for markerless deletion of CCNA_01217 & This study \\
\hline pEK406 & pVCHYC-5-based complementing vector, vanA::CCNA_01217-FLAG & This study \\
\hline pKR438 & pVGFPC-4-based complementing vector, vanA::CCNA_01217-FLAG & This study \\
\hline pKR429 & pNPTS138-based plasmid for markerless deletion of CCNA_01218 & This study \\
\hline pKR432 & CCNA_01218::FLAG in pFLGC-1 & This study \\
\hline pKR435 & pVGFPC-2-based complementation vector, vanA::CCNA_01218-FLAG & This study \\
\hline pGS76 & pNPTS138-based plasmid for markerless deletion of CCNA_01219 & This study \\
\hline pKR433 & CCNA_01219::FLAG in pFLGC-1 & This study \\
\hline pKR436 & pVGFPC-2-based complementation vector, vanA::CCNA_01219-FLAG & This study \\
\hline pEK722 & pNPTS138-based plasmid for markerless deletion of CCNA_01220 & $\begin{array}{r}\text { (Stankeviciute } \\
\text { et al., 2019) }\end{array}$ \\
\hline pKR434 & CCNA_01220::FLAG in pFLGC-1 & This study \\
\hline pKR437 & pVGFPC-2-based complementation vector, vanA::CCNA-01220-FLAG & This study \\
\hline pKR438 & pVGFPC-4-based complementation vector, vanA::CCNA-01217-FLAG & This study \\
\hline
\end{tabular}

\section{8}


Table S6, related to STAR Methods: Primers used in this study.

\begin{tabular}{|c|c|}
\hline Name & Sequence (5'-3') \\
\hline pJS14-PxylX & AGAACTAGTGGATCCTCACATGGTCTCGAA \\
\hline PxylX-IpxC R & ACCCGAAGCCGACACGGCGTCGTCTCCCCA \\
\hline PxylX-IpxC F & TGGGGAGACGACGCCGTGTCGGCTTCGGGT \\
\hline IpxC-3xFLAG R & GTAGTCCATGGATCCAACCGCTTCTGCAAG \\
\hline IpxC-3xFLAG F & CTTGCAGAAGCGGTTGGATCCATGGACTAC \\
\hline ssrA-pJS14 & CTTGATATCGAATTCTCACGCAGCGACGGC \\
\hline pVCERN-2 $00497 \mathrm{~F}$ & ACGCATATGAACAGCATTCTCCCG \\
\hline pVCERN-2 00497 R & CCGGAGCTCCTAGATCGGCCGGCC \\
\hline pVCERN-2 $01553 \mathrm{~F}$ & ACGCATATGAAGCGTATGTTTGAT \\
\hline pVCERN-2 $01553 \mathrm{R}$ & CCGGAGCTCCTAACGGGTGACGCC \\
\hline pVCERN-2 $03733 \mathrm{~F}$ & ACGCATATGGCTGCGATCTATCCG \\
\hline pVCERN-2 $03733 \mathrm{R}$ & CCGGAGCTCTCAACGCGGCTTCGT \\
\hline Pvan-fur & GAGGAAACGCATATGGATCGACTCGAAAAG \\
\hline fur-pVCERN & AATTCTCCGGAGCTCTTACTCCTCCAGCGG \\
\hline 3xFLAG-F & cgccggcggatccatggactacaaag \\
\hline 3xFLAG-JS14 & CTTGATATCGAATTCTCACTTGTCATCGTCATC \\
\hline 00497::hyg UpF & CTCACTAGTAGGACGACGCCCATA \\
\hline 00497::hyg UpR & ATCCCCGGGGGCAAGGGTCGAGAC \\
\hline 00497::hyg DownF & ATCCCCGGGCGCCCGCTGCTGTGG \\
\hline 00497::hyg DownR & AGCGAATTCACCGAGGATTTGGTT \\
\hline 01055::hyg UpF & CTCACTAGTGCTGGCGCTGGAAGA \\
\hline 01055::hyg UpR & ACCGGATCCTTGAGCGCCATGGGC \\
\hline 01055::hyg DownF & ACCGGATCCGATGGACGAGCGCAG \\
\hline 01055::hyg DownR & AGCGAATTCTACGATGACGAGTCG \\
\hline 01068::hyg UpF & CTCACTAGTGCGCGAGGACACCGT \\
\hline 01068::hyg UpR & ACCGGATCCGACGCCTGGGTGGCG \\
\hline 01068::hyg DownF & ACCGGATCCCAGGCGGCGCCATAT \\
\hline 01068::hyg DownR & AGCGAATTCTTGACCTGCTTGAGC \\
\hline $01553 \mathrm{UpF}$ & AAAACTAGTATCGAGCAGGGCGTC \\
\hline 01553 UpR & GGCCCCGGGATCAAACATACGCTT \\
\hline 01553 DownF & ATCCCCGGGCCGGTGCTGACCGCA \\
\hline 01553 DownR & AAAGAATTCTATGCCGCCAAGCT \\
\hline 03733::hyg UpF & CTCACTAGTGACGCTGGCCCTTGT \\
\hline 03733::hyg UpR & ACCGGATCCGAAGGCGTGATCGAG \\
\hline
\end{tabular}




\begin{tabular}{|c|c|}
\hline 03733::hyg DownF & ACCGGATCCGCCGCCACACAGGAT \\
\hline 03733::hyg DownR & AGCGAATTCATGCTCAAGGACCTC \\
\hline fur UpF & CTCACTAGTAAGAGGGTGACCTCG \\
\hline fur UpR & ACCGGATCCGAGCTCTACGGGATG \\
\hline fur DownF & ACCGGATCCTTCGATACAGGCCTT \\
\hline fur DownR & AGCGAATTCTATATGCAGGCCTTC \\
\hline IpxC KO F & CTTCGAAACGGCCGATGATG \\
\hline IpxC KO R & GATCAACAACGCCGATGACG \\
\hline IpxC UpF & CTCACTAGTTCAGATAGGCTTCGA \\
\hline IpxC UpR & CATGAATTCCTCAATAACGCCGTG \\
\hline IpxC DownF & CTTGAATTCCGTGTGCTGAAAATA \\
\hline IpxC DownR & GACGCATGCTGGCCGCAAGCCGCG \\
\hline aacC1 EcoRI F & CGCGAATTCgaattgacataagcc \\
\hline aacC1 EcoRI R & GGGGAATTCgaattggccgcggcg \\
\hline ctpA KO F & GAAGAAGCGCGGGATCAAGA \\
\hline ctpA KO R & GTTGCCATGCTTGATGTGCA \\
\hline EK897 & AAGCTTGGCGCCAGCCGG \\
\hline EK898 & GAATTCGCTAGCTTCGGC \\
\hline EK1047 & gctggcgccaagcttAGGCGCTCGATCTGATCTTG \\
\hline EK1048 & caggaagatCGGCCCTTTTTGAACTTCAC \\
\hline EK1049 & aagggccgATCTTCCTGGCCCTCTTC \\
\hline EK1050 & cgaagctagcgaattcCTTGATCGTCGGGTTCTC \\
\hline EK1055 & gctggcgccaagcttGGCTTCTTCCACAACTTTG \\
\hline EK1056 & accttgagGAGGACCCCGGACATATC \\
\hline EK1057 & gggtcctcCTCAAGGTCTGGAGAGAG \\
\hline EK1058 & cgaagctagcgaattcCGTCATCTGTCGTCCCTAC \\
\hline 01218 up_fwd & attgaagccggctggcgccaTGGCACGGCCATTTCGGC \\
\hline 01218 up_rev & tgagcccaaaGCCGTCGATCACCAGCAAG \\
\hline 01218 down_fwd & gatcgacggcTTTGGGCTCAAGCCGTTCG \\
\hline 01218 down_rev & cgtcacggccgaagctagcgGCCGTTCGACAAGCCGCG \\
\hline EK1357 & tactcatATGAGTAGTGAAGTTCAAAAAGGGCCG \\
\hline EK1358 & tactgctagcTTActtgtcatcgtcatccttgtagtcTTTCGCCAGCCAGGACTGG \\
\hline EK S216 & ACCCCGTCTGATAAGGCTTC \\
\hline EK S217 & GCGAGACCGTGATCGACT \\
\hline EK S238 & GTAGGCAGGGTCCGACAGT \\
\hline EK S239 & ACCGCAAAGTTGTGGAAGAA \\
\hline
\end{tabular}




\begin{tabular}{|c|c|}
\hline EK S240 & ATGACCTTCCTCGACACG \\
\hline EK S241 & GAGTCGATCACGGTCTCG \\
\hline EK S242 & AGGGCTTCTTCTTTGGCATT \\
\hline EK S243 & ACTTCATCGTCGGCACCTT \\
\hline RecUni-1 & ATGCCGTTTGTGATGGCTTCCATGTCG \\
\hline RecVan-2 & CAGCCTTGGCCACGGTTTCGGTACC \\
\hline Nde-01218 & TATATTCATATGCTTCGTCGTGCACGCCATCC \\
\hline 01218-Mlu & ATTATACGCGTTCCGACCAGGAACCGCAAGGC \\
\hline Nde-01219 & TATATTCATATGAGCCGCCTGCGCGGCCTC \\
\hline 01219-Mlu & TAATAACGCGTTGCGGCTTGCCGCCGCTCTC \\
\hline Nde-01220 & TATATTCATATGGGGCTATTTGATAAGCACCTGGCC \\
\hline 01220-Mlu & AATAACGCGTGGCGCGGGCGCGCTTGAG \\
\hline 01217-FLAG F & CCACGATGCGAGGAAACGCATATGAGTAGTGAAGTTCAAAAAG \\
\hline 01217-FLAG R & AATTAAGGCGCCTGCAGGCAGCTAGCTCACTTGTCATC \\
\hline 01218-FLAG F & CCACGATGCGAGGAAACGCATATGCTTCGTCGTGCACG \\
\hline 01218-FLAG R & AATTAAGGCGCCTGCAGGCAGCTAGCTTACTTGTCATCGTC \\
\hline 01219-FLAG F & CCACGATGCGAGGAAACGCATATGAGCCGCCTGCGCGG \\
\hline 01219-FLAG R & AATTAAGGCGCCTGCAGGCAGCTAGCTTACTTGTCATCGTCATCCTTGTAG \\
\hline 01220-FLAG F & CCACGATGCGAGGAAACGCATATGGGGCTATTTGATAAG \\
\hline 01220-FLAG R & AATTAAGGCGCCTGCAGGCAGCTAGCTTACTTGTCATC \\
\hline
\end{tabular}

1450 

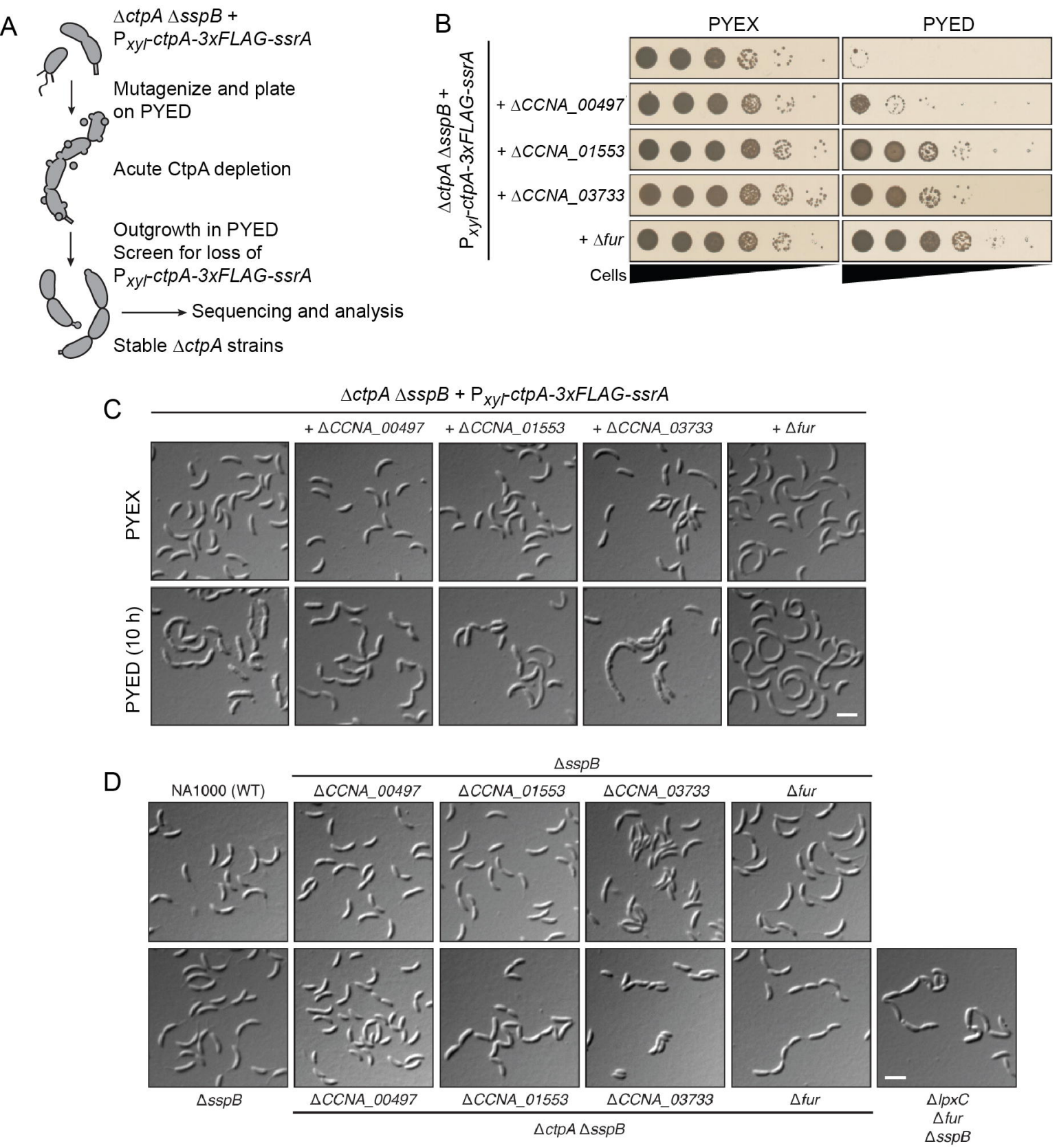
Figure 2

$\mathrm{A}$
$\mathrm{B}$
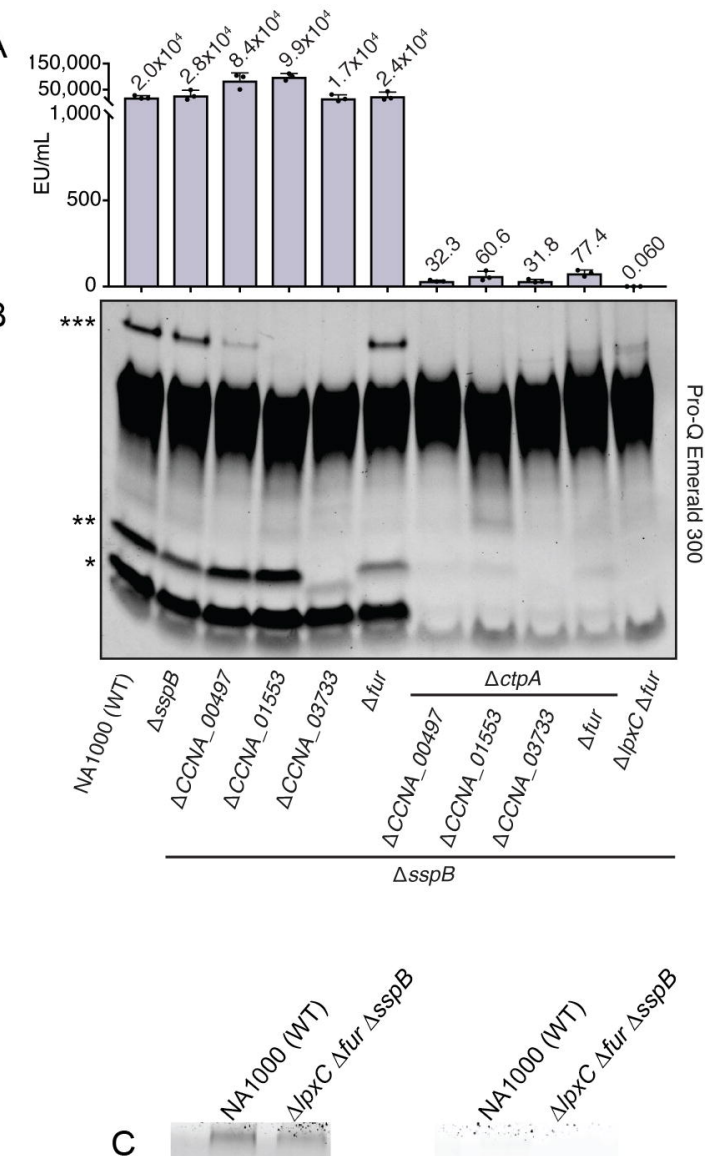

C

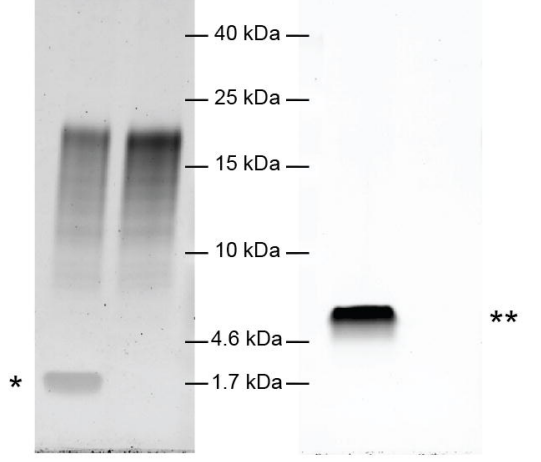

D

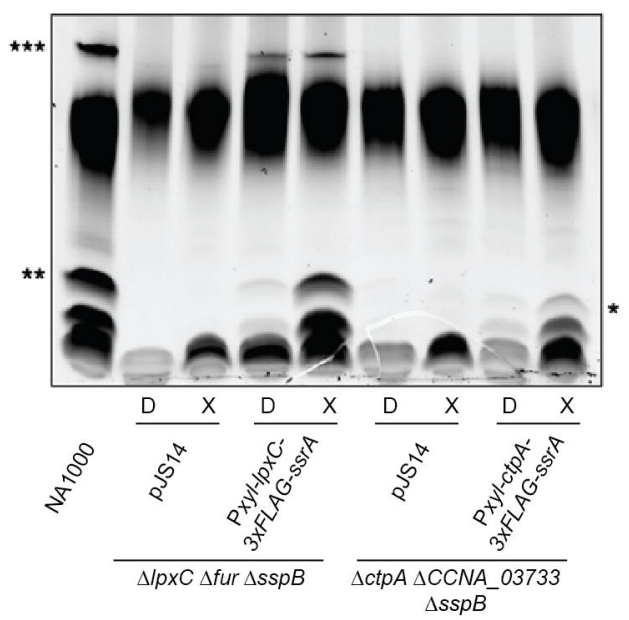

$\triangle c t p A \Delta s s p B+$

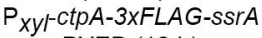

E
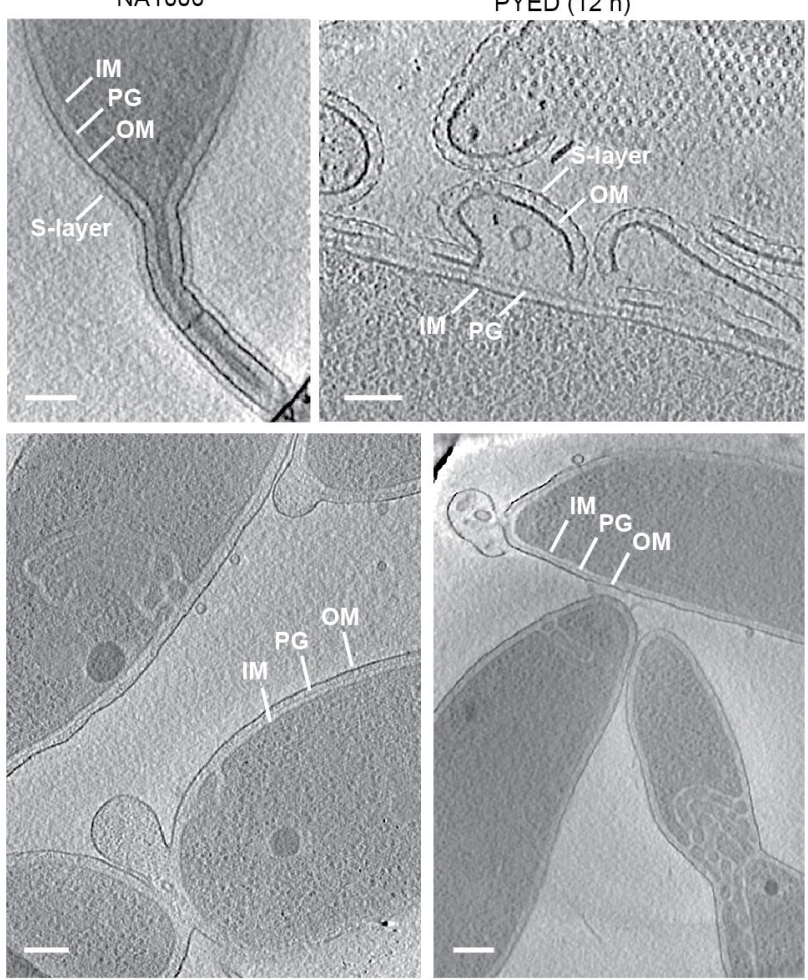

$\Delta c t p A \Delta f u r \Delta s s p B$

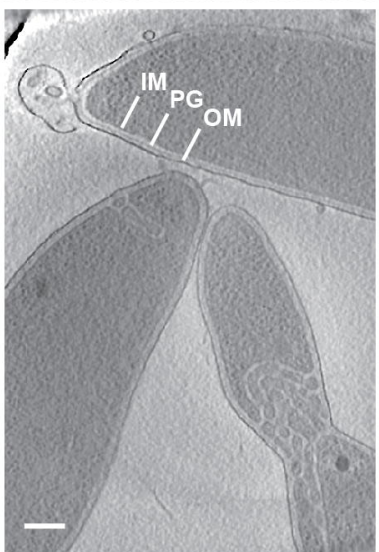

$\Delta / p \times C \Delta f u r \Delta s s p B$ 
Figure 4
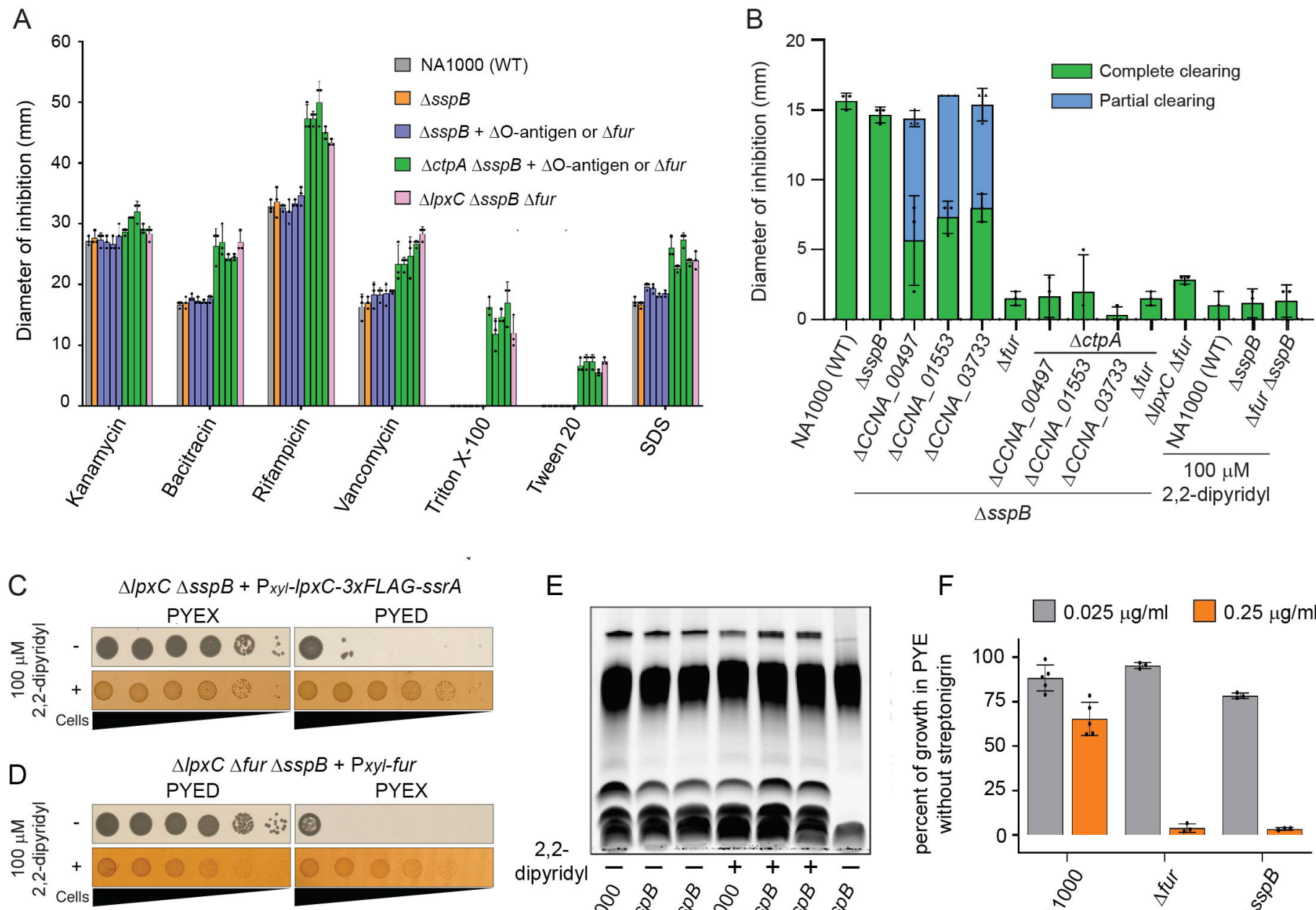

E

2,2dipyridyl

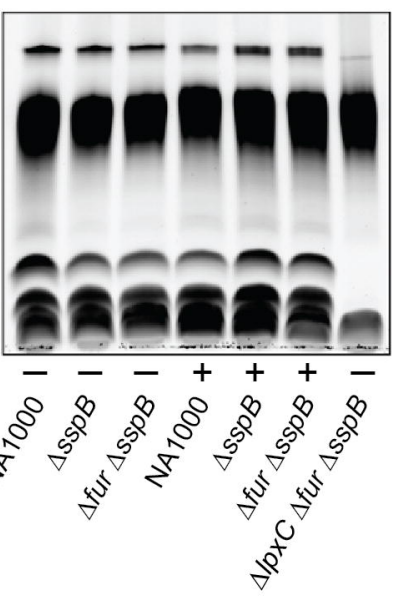

F $\square 0.025 \mu \mathrm{g} / \mathrm{ml} \quad \square 0.25 \mu \mathrm{g} / \mathrm{ml}$

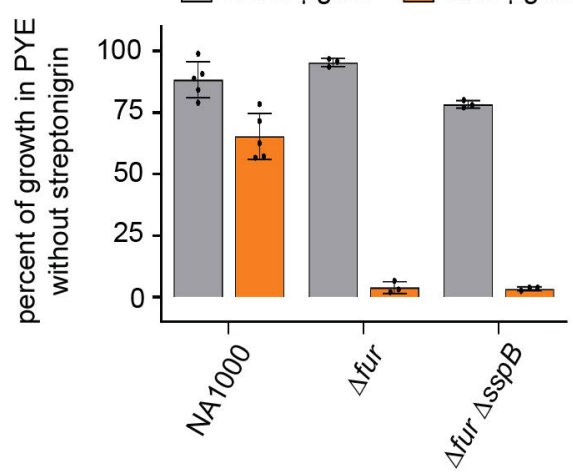

G

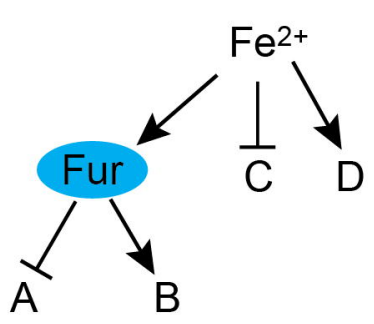

Wild-type lipid A essential

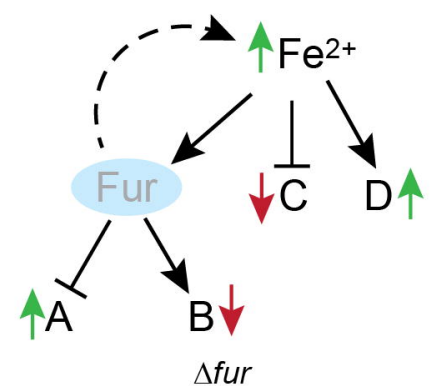

lipid A dispensable

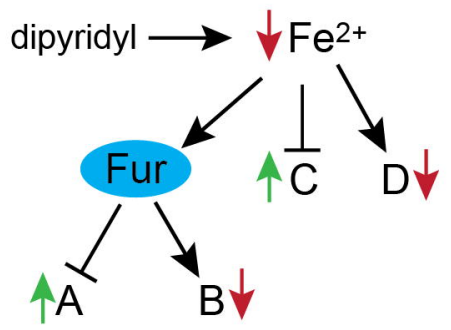

Wild-type + dipyridyl lipid A dispensable 

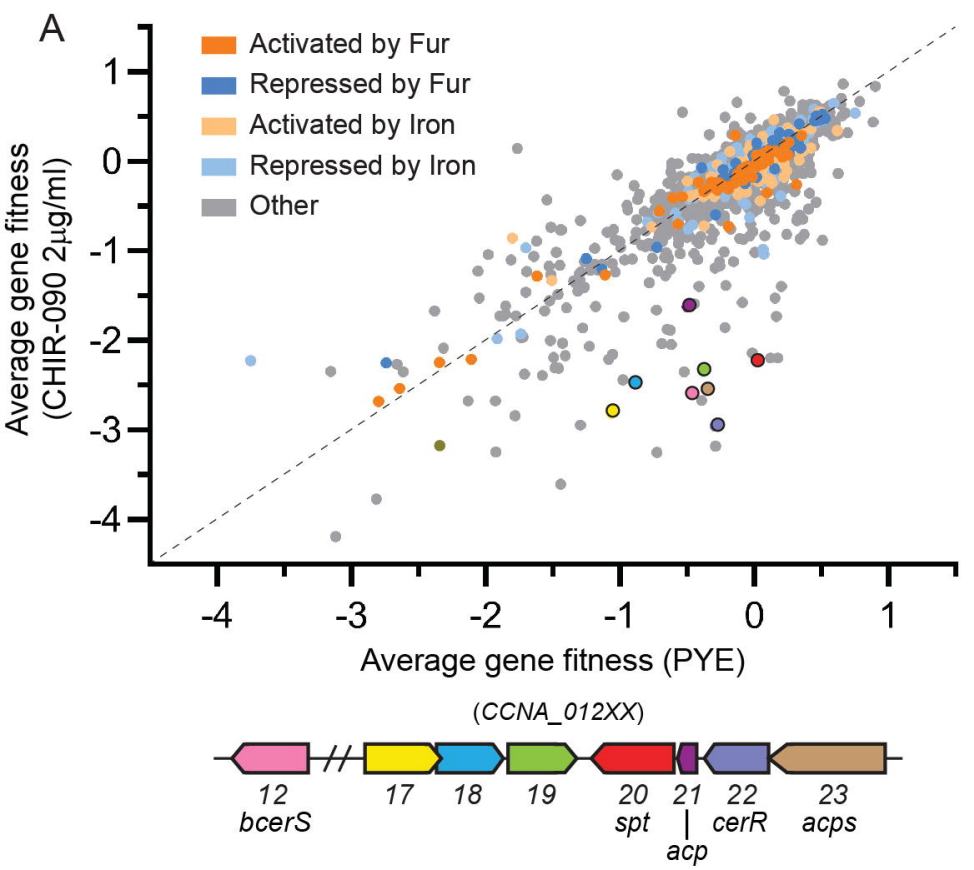

B
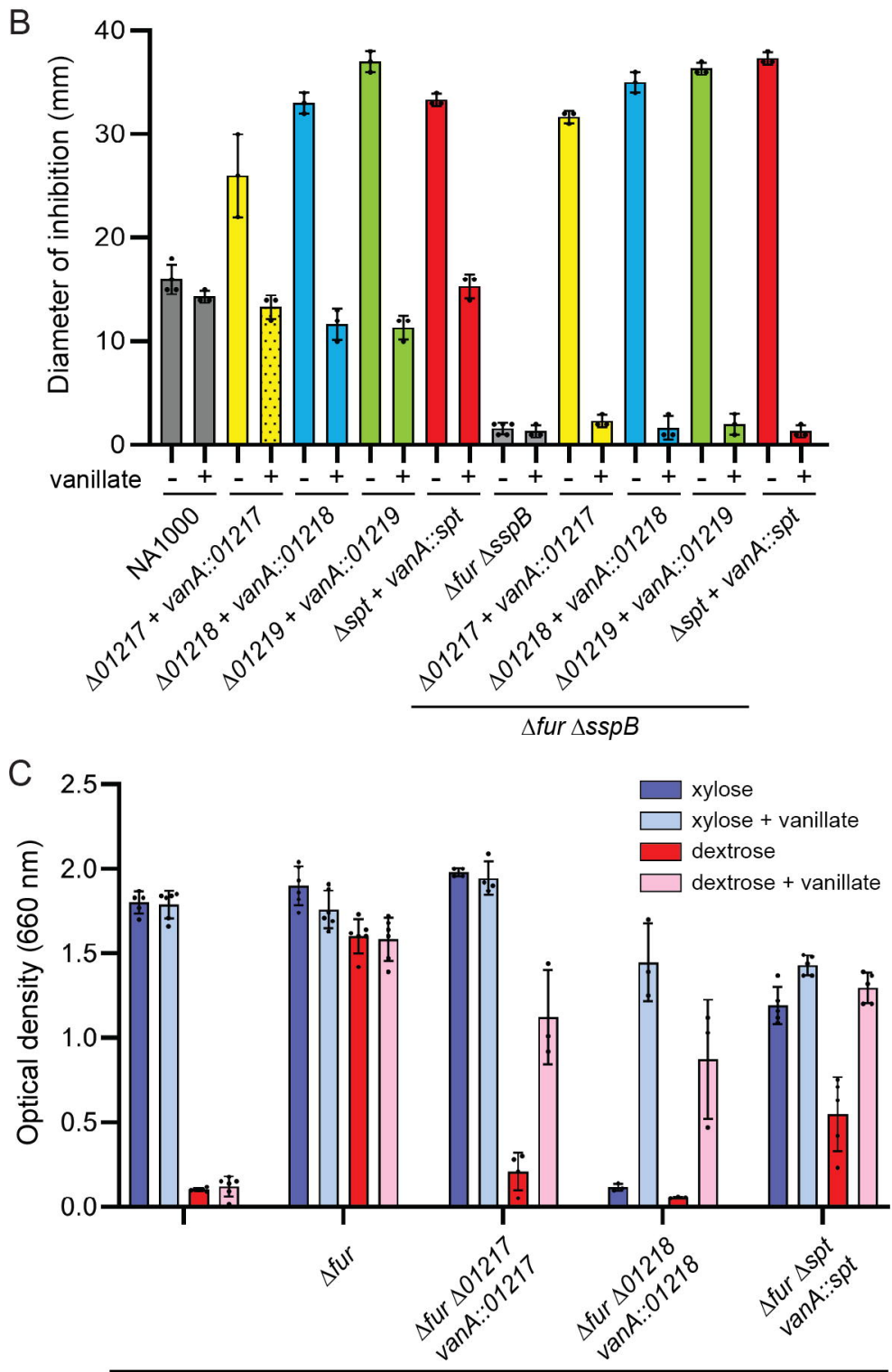


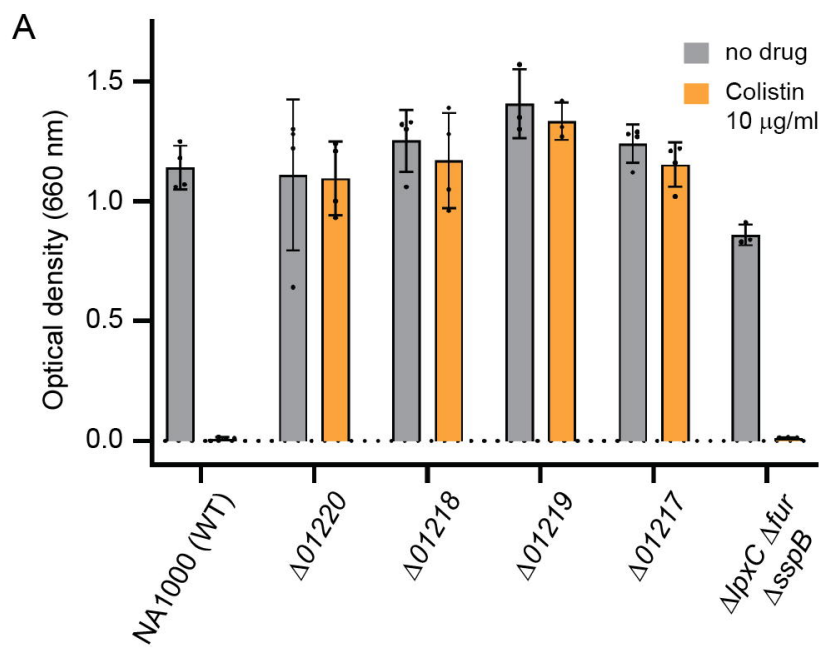

Outer

B

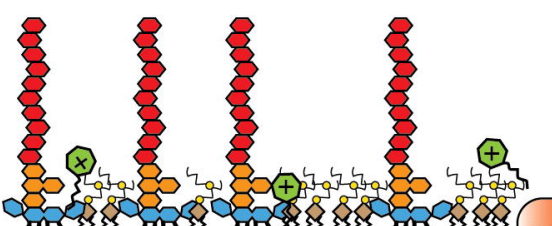

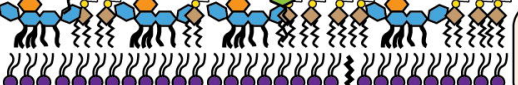

Lipoproteins

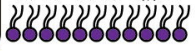

Peptido-

glycan

Periplasm

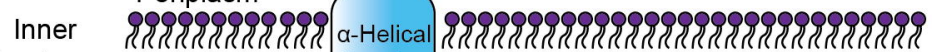

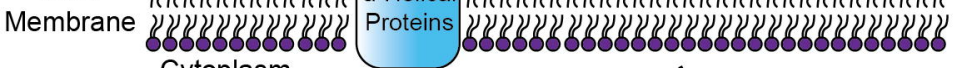
Cytoplasm

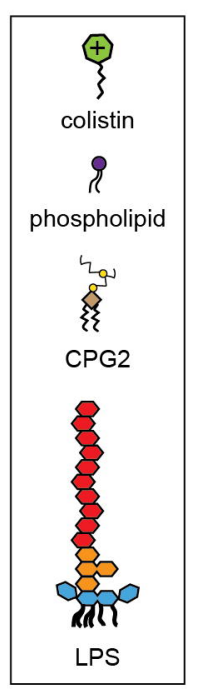
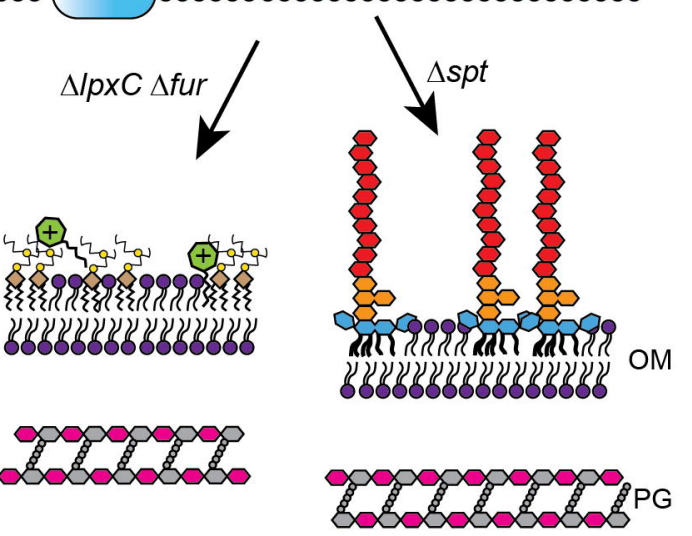

IM

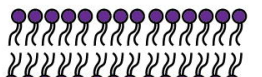

wild-type colistin sensitivity

रदรर

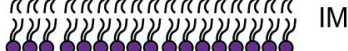

reduced colistin sensitivity 